\title{
THE ACCURACY OF INTERPOLATION BY FINITE DIFFERENCES
}

\author{
(Second Paper)
}

\author{
By W. F. Sheppard.
}

\begin{abstract}
[Received March 29th, 1910.-Read April 28th, 1910.-Received in revised form, May 11th, 1911.]
\end{abstract}

Note.-This is a continuation of a paper with the same title in Proceedings of the London Mathematical Society, Series 2, Vol. 4, pp. 320-341. For convenience of reference, the numbers of the sections and of the formulæ in the two papers are made consecutive. The table on p. 331 of the first paper is quoted as Table I.

The following is a summary of the contents of the two papers:-

Introductory $(\S 1)$

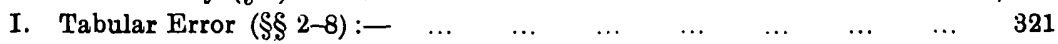

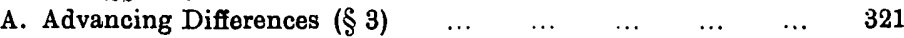

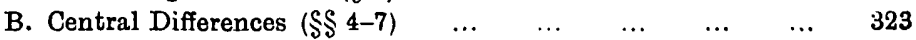

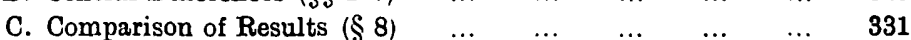

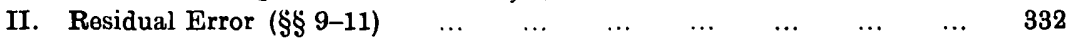

$\begin{array}{lllllllllll}\text { Appendix... } & \ldots & \ldots & \ldots & \ldots & \ldots & \ldots & \ldots & \ldots & \ldots & 339\end{array}$

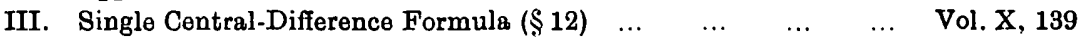

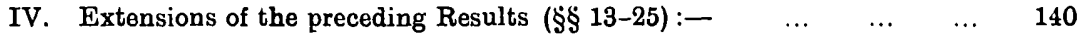

A. Formulæe comprised in the Lagrange-formula $(\$ \S 13-17) \quad \ldots \quad 140$

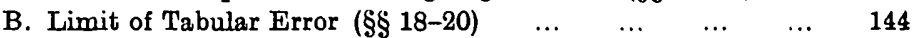

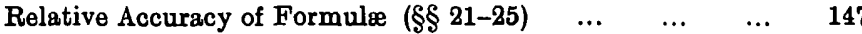

VI. The Arrangement of Mathematical Tables for Interpolation $\left(\begin{array}{llll}\S 36) & \ldots & 170\end{array}\right.$

\section{Single Central-Difference Formula.}

12. For central-difference interpolation, the standard formula has [ $\$ 5]$ been given in the form (16) for $0<\theta \leqslant \frac{1}{2}$, and in the form (17) for $-\frac{1}{2} \leqslant \theta<0$. It is usually convenient to take the interval $0<\theta<1$ as our basis. For the first half of the interval we have (16). For $\frac{1}{2}<\theta<1$ we have $-\frac{1}{2}<\theta-1<0$, so that the suitable formula is (17) adapted, viz.,

$u_{\theta}=u_{1+(\theta-1)}=u_{1}+\frac{\theta-1}{1 !} \delta u_{3}+\frac{\theta(\theta-1)}{2 !} \delta^{2} u_{1}+\frac{\theta(\theta-1)(\theta-2)}{3 !} \delta^{8} u_{3}+\ldots$ 
If we compare this formula and (16) and (17) with each other, we see that (16) and (17) are identical if we take an odd number of terms, while (16) and (58) are identical if we take an even number of terms. If, however, (17) is the suitable formula, but the number of terms is even, or, if (58) is the suitable formula, but the number of terms is odd, we can still use (16), provided that in the final term we substitute the proper central difference without altering its coefficient.

We can therefore regard (16) as the standard formula either for the whole interval $\theta=-\frac{1}{2}$ to $\theta=+\frac{1}{2}$ or for the whole interval $\theta=0$ to $\theta=1$, provided that, (i) if $-\frac{1}{2} \leqslant \theta<0$, and we go up to $\delta^{2 u+1} u$, we replace $\delta^{2 n+1} u_{\mathrm{j}}$ by $\delta^{2 n+1} u_{-\frac{1}{2}}$, and (ii), if $\frac{1}{2}<\theta<1$, and we go up to $\delta^{2 n} u$, we replace $\delta^{2 n} u_{0}$ by $\delta^{2 n} u_{1}$.

It may, however, be mentioned that, in the second of these two cases, the correction need not be made if we are only considering the tabular error; i.e., the failure to make the correction, although it prevents the formula used from being the true central-difference formula, does not increase the maximum-limit of the tabular error [see $\$ 23$ (ii)].

\section{Extensions of the preceding Results.}

A. Formula comprised in the Lagrange-formula.

13. In $\$ 9$ the advancing-difference and central-difference expressions for $u_{\theta}$, taken up to the difference of $m$-th order, were converted into expressions in terms of a series of $u$ 's; and it was found that these expressions were substantially of the same form. They are, in fact, the expressions given by Lagrange's interpolation-formula, as modified by the condition that the tabulated values of $x$ proceed by equal intervals; the values of $u$ which enter into the expression being in the one case $u_{0}, u_{1}, u_{2}, \ldots$ if $\theta$ lies between 0 and 1 , while in the other case, if

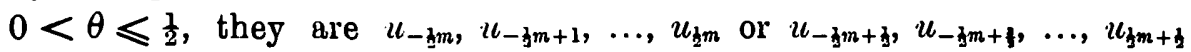
according as $m$ is even or odd. Also, we see from $\$ 12$ that the centraldifference formula for $\frac{1}{2} \leqslant \theta<1$ leads to the same expression if $m$ is odd, while, if $m$ is even, it gives a similar expression involving

$$
u_{-\frac{1}{2} m+1}, u_{-\frac{1}{2} m+2}, \ldots, u_{t_{2} m+1} .
$$

If therefore we write Lagrange's formula in the form

where

$$
u_{\theta}={ }_{m} L_{\theta-p},
$$

$$
\begin{aligned}
{ }_{m} L_{\theta-p} \equiv \frac{(\theta-p)(\theta-p-1) \ldots(\theta-p-m)}{m !} & \\
& \times\left\{\frac{u_{p+m}}{\theta-p-m}-{ }_{m} C_{1} \frac{u_{p+m-1}}{\theta-p-m+1}+\ldots+(-)^{m} \frac{u_{p}}{\theta-p}\right\},
\end{aligned}
$$


then, in the notation of $\S 9$, the advancing-difference formula is

$$
u_{\theta}={ }_{m} U_{\theta}={ }_{m} L_{\theta} \quad(0<\theta<1),
$$

while the central-difference formula takes the different forms

$$
\begin{array}{cc}
u_{\theta}={ }_{2 n} V_{\theta}={ }_{2 n} L_{\theta+n} & \left(0<\theta \leqslant \frac{1}{2}\right), \\
u_{\theta}={ }_{2 n} V_{\theta-1}={ }_{2 n} L_{\theta+n-1} & \left(\frac{1}{2} \leqslant \theta<1\right), \\
u_{\theta}={ }_{2 n+1} V_{\theta}={ }_{2 n+1} L_{\theta+n} & (0<\theta<1) .
\end{array}
$$

14. Conversely, if we start with Lagrange's formula $u_{\theta}={ }_{n} L_{\theta-p}$, we can, by giving $p$ a suitable value, convert ${ }_{m} L_{\theta-p}$ into any one of the above expressions, such as ${ }_{m} U_{\theta}$, by working backwards and taking out differences of the $m$-th, $(m-1)$-th, $\ldots$ orders in succession ; the number of $u$ 's in the remainder being reduced at each step. It is obvious, however, that, when the value of $p$ has been settled, this process of taking out differences can be performed in a good many different ways besides the particular one which leads to the expression we are considering, and that we shall thus arrive at other formulæ which will be equivalent to, and might be substituted for, the advancing-difference or the central-difference formula, as the case may be. Denote the difference of order $n$, which involves $u_{q}, u_{q+1}, \ldots, u_{q+n}$, by $[q, q+1, \ldots, q+n]$, so that $[q, q+1, \ldots, q+n]$

$$
\equiv \Delta^{n} u_{q}=\delta^{n} u_{q+\frac{1}{n}}=u_{q+n}-{ }_{n} C_{1} u_{q+n-1}+\ldots+(-)^{n} u_{q} .
$$

Then from ${ }_{m} L_{\theta-p}$ we can take out $[p, p+1, \ldots, p+m]$ in either of two ways, according as we are to get rid of $u_{p+m}$ or of $u_{p}$; its coefficient will in the former case be $(\theta-p)(\theta-p-1) \ldots: \theta-p-m+1) / m$ !, and in the latter case $(\theta-p-1)(\theta-p-2) \ldots(\theta-p-m) / m$ !. Similarly, supposing we get rid of $u_{p+m}$, we can at the next nove get rid either of $u_{p+m-1}$ or of $u_{r}$. Proceeding in this way, we see that there are $2^{m}$ different expressions of this kind which would produce the same expression ${ }_{m} L_{\theta-p}$; these expressions corresponding to the different ways by which we can, in the first diagram in $\$ 4$, proceed from $\Delta^{n} u_{p}$ to some value of $u$ by steps, each of which is from a quantity in one column of the diagram to one of the two adjoining quantities in the preceding column. Of these $2^{m}$ expressions, the numbers which contain $u_{n}, u_{p+1}, u_{p+2}, \ldots, u_{p+m}$ are respectively $1,{ }_{m} C_{1},{ }_{m} C_{2}, \ldots, 1$.

If in the diagram mentioned above we draw lines diagonally upwards and downwards to the left, so as to include $\Delta^{m} u_{p}$ but no other differences of the $m$-th order, and so as to meet the line which divides the column of $u$ 's from the column of $x$ 's, all the tabulated quantities which occur in any one of the equivalents of ${ }_{m} L_{\theta-p}$ will lie within the triangle so formed. This will be called the tabular triangle of $\Delta^{m} u_{p}$. 
15. We have next to consider the law of formation of the successive terms in any one of these expressions, proceeding from the left. The law is most easily determined by working backwards.

(i) As shown in $\$ 14$, we can remove from ${ }_{m} L_{\theta-p}$ either

$$
\{(\theta-p)(\theta-p-1) \ldots(\theta-p-m+1) / m !\}[p, p+1, \ldots, p+m]
$$

or $\quad\{(\theta-p-1)(\theta-p-2) \ldots(\theta-p-m) / m !\}[p, p+1, \ldots, p+m]$ s

It will be found that in the one case we are left with ${ }_{m-1} L_{\theta-p}$ and in the other with ${ }_{n-1} L_{\theta-p-1}$. Hence we have

${ }_{m} L_{\theta-p}={ }_{m-1} L_{\theta-p}+\frac{(\theta-p)(\theta-p-1) \ldots(\theta-p-m+1)}{m !}[p, p+1, \ldots, p+m]$,

${ }_{m} L_{\theta-r}$

$={ }_{m-1} L_{\theta-p-1}+\frac{(\theta-p-1)(\theta-p-2) \ldots(\theta-p-m)}{m !}[p, p+1, \ldots, p+m]$.

(ii) Hence the removal of successive terms, from the right, will always leave us with an expression of the Lagrange form; and, conversely, any number of terms, from the left, will together give an expression of this form. Also ${ }_{m-1} L_{\theta-p}$ contains $u_{p}, u_{p+1}, \ldots, u_{p+m-1}$, and ${ }_{m-1} L_{\theta-p-1}$ contains $u_{p+1}, u_{p+2}, \ldots, u_{p+m}$. We see therefore, from (66) and (67), that the first $n+1$ terms will together be equal to an expression of the form " $L_{\theta-4}$, involving $u_{1}, u_{q+1}, \ldots, u_{q+n}$; that the next term to be added will be either

or

$$
\begin{aligned}
& \frac{(\theta-q)(\theta-q-1) \ldots(\theta-q-n)}{(n+1) !}[q, q+1, \ldots, q+n+1] \\
& \frac{(\theta-q)(\theta-q-1) \ldots(\theta-q-n)}{(n+1) !}[q-1, q, \ldots, q+n] ;
\end{aligned}
$$

and that the result of the addition will be in the one case ${ }_{n+1} L_{\theta-q}$, and in the other case ${ }_{n+1} L_{\theta-q+1}$.

(iii) Instead of regarding each coefficient as a whole, we may regard it as made up of successive factors. Continuing the terms in (ii), the coefficient of the difference of order $n+2$ will in the one case be

$$
\begin{aligned}
& \frac{(\theta-q)(\theta-q-1) \ldots(\theta-q-n-1)}{(n+2) !} \\
& =\frac{(\theta-q)(\theta-q-1) \ldots(\theta-q-n)}{(n+1) !} \frac{\theta-(q+n+1)}{n+2},
\end{aligned}
$$

and in the other case will be

$\frac{(\theta-q+1)(\theta-q) \ldots(\theta-q-n)}{(n+2) !}=\frac{(\theta-q)(\theta-q-1) \ldots(\theta-q-n)}{(n+1) !} \frac{\theta-(q-1)}{n+2}$. 
Hence (changing $n+2$ into $r$ ), if the $r$-th term introduces $u_{s}$, the coefficient of the difference in the $(r+1)$-th term will be found by multiplying the coefficient of the difference in the $r$-th term by $(\theta-s) / r$.

(iv) We may take as examples the advancing-difference formula (3) and the central-difference formulæ (16), (17), and (58), which may be written

$$
\begin{aligned}
& u_{\theta}=[0]+\frac{\theta-0}{1}[0,1]+\frac{\theta-0}{1} \frac{\theta-1}{2}[0,1,2] \\
& +\frac{\theta-0}{1} \frac{\theta-1}{2} \frac{\theta-2}{3}[0,1,2,3]+\ldots, \\
& u_{\theta}=[0]+\frac{\theta-0}{1}[0,1]+\frac{\theta-0}{1} \frac{\theta-1}{2}[-1,0,1] \\
& +\frac{\theta-0}{1} \frac{\theta-1}{2} \frac{\theta-(-1)}{3}[-1,0,1,2]+\ldots, \\
& u_{\theta}=[0]+\frac{\theta-0}{1}[-1,0]+\frac{\theta-0}{1} \frac{\theta-(-1)}{2}[-1,0,1] \\
& +\frac{\theta-0}{1} \frac{\theta-(-1)}{2} \frac{\theta-1}{3}[-2,-1,0,1]+\ldots, \\
& u_{\theta}=[1]+\frac{\theta-1}{1}[0,1]+\frac{\theta-1}{1} \frac{\theta-0}{2}[0,1,2] \\
& +\frac{\theta-1}{1} \frac{\theta-0}{2} \frac{\theta-2}{3}[-1,0,1,2]+\ldots .
\end{aligned}
$$

16. It follows from the above that we need not define the centraldifference formula of order $m$ (i.e., up to and including a difference of that order) for $u_{\theta}$ otherwise than either (i) as a formula which is algebraically equivalent to the Lagrange-formula involving $u_{p}, u_{p+1}, \ldots, u_{p+m}$, where $p$ is given by the condition that $x_{p+k m-k} \leqslant x_{\theta} \leqslant x_{p+m n+k}$, or (ii) as a formula expressing $u_{\theta}$ as a series constructed in the manner explained in $\S 15$ and ending with that difference of the $m$-th order which lies nearest to the horizontal line through the point corresponding to the value of $x_{\theta}$.

17. We can, of course, express ${ }_{m} L_{\theta-p}$ in a very large number of ways in terms of the quantities lying within the tabular triangle of $\Delta^{m} u_{p}$. Everett's formula, for instance, is a variant in which $m$ is odd, and each difference of odd order is expressed in terms of the adjoining differences of the next lower order.

It will be convenient here to examine the effect of applying this process to a single term in the more general formula considered above. 
Suppose that we have a term in $\Delta^{n} u_{y}$, and that we replace $\Delta^{n} u_{\eta}$ by $\Delta^{n-1} u_{\eta+1}-\Delta^{n-1} u_{q}$; then the result will depend on the steps by which we have reached $\Delta^{n} u_{\eta}$ from a difference of order $n-2$, but it will be found that the two cases, in each of which one step is by advancing and the other by receding, will give the same result. (This is obvious on general grounds; for the terms in $\Delta^{n-1} u_{\eta l}$ and in $\Delta^{n-1} u_{q+1}$ are in each case together equal to ${ }_{n} L_{\theta-q}-{ }_{n-2} L_{\theta-\eta-1}$, and their coefficients must therefore be the same in the two cases.) There are therefore three formula.

(i) Where $\Delta^{n-2} u_{q}$ is involved,

$$
\begin{aligned}
{ }_{n} L_{\theta-\imath}={ }_{n-2} L_{\theta-4} & +\frac{(\theta-q)(\theta-q-1) \ldots(\theta-q-n+2)}{n !} \\
& \times\left\{(\theta-q-n+1) \Delta^{n-1} u_{\eta+1}-(\theta-q-2 n+1) \Delta^{n-1} u_{q}\right\} .
\end{aligned}
$$

(ii) Where $\Delta^{n-2} u_{q+1}$ is involved,

$$
\begin{aligned}
{ }_{n} L_{\theta-q}={ }_{n-2} L_{\theta-q-1} & +\frac{(\theta-q-1)(\theta-q-2) \ldots(\theta-q-n+1)}{n !} \\
& \times\left\{(\theta-q) \Delta^{n-1} u_{q+1}-(\theta-q-n) \Delta^{n-1} u_{q}\right\} .
\end{aligned}
$$

(iii) Where $\Delta^{n-2} u_{q+2}$ is involved,

$$
\begin{aligned}
{ }_{n} L_{\theta-4}={ }_{n-2} L_{\theta-q-2}+ & \frac{(\theta-q-2)(\theta-q-8) \ldots(\theta-q-n)}{n !} \\
& \times\left\{(\theta-q+n-1) \Delta^{n-1} u_{q+1}-(\theta-q-1) \Delta^{n-1} u_{q}\right\} .
\end{aligned}
$$

B. Limit of Tabular Error.

18. In $\$ \S 3$ and 6 we considered the limits of tabular error for the advancing-difference and the central-difference formulæ respectively. We have now to consider the limit for the more general formula, i.e., we have to consider the limit of error involved in calculating the general expression ${ }_{i n} L_{\theta-p}$, for the purpose of (59), from the tabulated values of $u$, instead of from the true values. It will be convenient to regard $\theta$ as lying between 0 and 1 , and $p$ as arbitrary; so that the cases already considered are those of $p=0$ and $p+\frac{1}{2} m-\frac{1}{2} \leqslant \theta \leqslant p+\frac{1}{2} m+\frac{1}{2}$, and we have now to regard $p$ as having any positive or negative integral value. It will be assumed throughout that the limits of error of each tabulated $u$ are $\pm \frac{1}{2} \rho$.

19. Let $a_{\eta}$, as before, denote the error of the tabulated value of $u_{q}$. Then the tabular error of $u_{\theta}$, as given by (59) or by any equivalent formula, is to be found by replacing each $u$ in ${ }_{n} L_{\theta-p}$ by the corresponding value of $\alpha$. Denote the result by ${ }_{m} A_{\theta-p}$. Then, for any given values of $\theta, m$, and $p,{ }_{m} A_{\theta-p}$ will have its numerically greatest value when each 
value of $\alpha$ is $+\frac{1}{2} \rho$ or $-\frac{1}{2} \rho$, the signs being such that the terms within the bracket in (60) all have the same sign. This requires that the $a^{\prime} s$ should be alternately positive and negative, except where the denominators in (60) change sign, in which case two consecutive a's must have the same sign. This, of course, will only happen when $\theta$ lies between $p$ and $p+m$, i.e., when $p \ngtr 0$ and $p+m \nless 1$. Hence, taking $\alpha_{0}$ to be positive, the sequence of values of $a$ which will make the tabular error a maximum (numerically) will, as in the particular cases already considered in $\$ \S 3$ and 6 , be

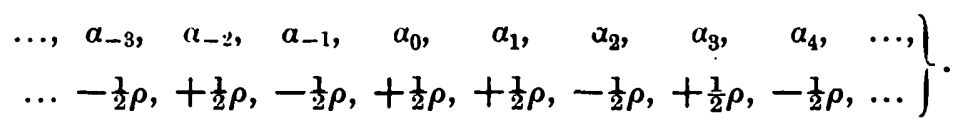

Denote these values of $a$ by $\ldots \epsilon_{-1}, \epsilon_{0}, \epsilon_{1}, \epsilon_{2}, \ldots$, and the resulting value of ${ }_{m} A_{\theta-p}$ by ${ }_{m} E_{\theta-p}$. Then, since ${ }_{m} E_{\theta-p}$ is an expression of the same form as ${ }_{n} L_{\theta-\eta}$, it follows that the tabular error due to using any formula which is equivalent to the Lagrange-formula may be obtained by applying this formula to the caiculation of a quantity $\epsilon_{\theta}$ from a table based on the above values, as follows :-

TABLE II.

\begin{tabular}{|c|c|c|c|c|c|c|c|c|c|}
\hline$x$ & $\epsilon$ & 1st Diff. & 2nd Diff. & 3rd Diff. & 4th Diff. & 5th Diff. & 6th Diff. & 7th Diff. & $\ldots$ \\
\hline $\begin{array}{c}\vdots \\
x_{-3} \\
x_{-2} \\
x_{-1} \\
x_{0} \\
x_{1} \\
x_{2} \\
x_{3} \\
x_{4} \\
\vdots\end{array}$ & $\begin{array}{c}\vdots \\
-\frac{1}{2} \rho \\
\frac{1}{2} \rho \\
-\frac{1}{2} \rho \\
\frac{1}{2} \rho \\
\frac{1}{2} \rho \\
-\frac{1}{2} \rho \\
\frac{1}{2} \rho \\
-\frac{1}{2} \rho \\
\vdots\end{array}$ & $\begin{array}{r}\rho \\
-\rho \\
\rho \\
0 \\
-\rho \\
\rho \\
-\rho \\
\vdots\end{array}$ & $\begin{array}{c}\vdots \\
2 \rho \\
-2 \rho \\
2 \rho \\
-\rho \\
-\rho \\
2 \rho \\
-2 \rho \\
2 \rho \\
\vdots\end{array}$ & $\begin{array}{c}\vdots \\
-4 \rho \\
4 \rho \\
-3 \rho \\
0 \\
3 \rho \\
-4 \rho \\
4 \rho \\
\vdots\end{array}$ & $\begin{array}{r}\vdots \\
-8 \rho \\
8 \rho \\
-7 \rho \\
3 \rho \\
3 \rho \\
-7 \rho \\
8 \rho \\
-8 \rho \\
\vdots\end{array}$ & $\begin{array}{c}\vdots \\
16 \rho \\
-15 \rho \\
10 \rho \\
0 \\
-10 \rho \\
15 \rho \\
-16 \rho \\
\vdots\end{array}$ & $\begin{array}{c}\vdots \\
32 \rho \\
-31 \rho \\
25 \rho \\
-10 \rho \\
-10 \rho \\
25 \rho \\
-31 \rho \\
32 \rho \\
\vdots\end{array}$ & $\begin{array}{c}\vdots \\
-63 \rho \\
56 \rho \\
-35 \rho \\
0 \\
35 \rho \\
-56 \rho \\
63 \rho \\
\vdots\end{array}$ & $\ldots$ \\
\hline
\end{tabular}

Suppose, for example, that $\theta=\cdot 2$, and that we are using the advancingdifference formula up to the 4 th difference inclusive. Then the limit of tabular error is

$$
\left\{\frac{1}{2} \rho+\frac{\cdot 2 \times \cdot 8}{2 !} \rho+\frac{\cdot 2 \times \cdot 8 \times 1 \cdot 8}{3 !} 3 \rho+\frac{\cdot 2 \times \cdot 8 \times 1 \cdot 8 \times 2 \cdot 8}{4 !} 7 \rho\right\}=\cdot 9592 \rho ;
$$

or, to three places of decimals, $\cdot 960 \rho$, as in Table 1 ( $(8)$.

sxr. 2. vor. 10 . No. 1107 . 
20. The following are sume simple properties of Table II and of the deduced values of $\epsilon_{\theta} \equiv{ }_{n} E_{\theta-1}$.

(i) (a) If $p+m \leqslant 0$, then $\Delta^{m} \epsilon_{p}=(-)^{p+m} 2^{m-1} \rho$.

(b) If $\quad p>0$, then $\left.\Delta^{m} \epsilon_{p}=(-)^{p+m-1} 2^{m-1} \rho.\right\}$

(ii) For the central-differences :-

$$
\begin{aligned}
\delta^{2 n-1} \epsilon_{1} & =0, \\
\delta^{2 n} \epsilon_{0} & =\delta^{2 n} \epsilon_{1}=(-)^{n}{ }_{2 n} C_{n} \cdot \frac{1}{2} \rho, \\
\mu \delta^{2 ! n} \epsilon_{\frac{1}{2}} & =(-)^{n}{ }_{2 n} C_{n} \cdot \frac{1}{2} \rho, \\
\mu \delta^{2 n-1} \epsilon_{0} & =(-)^{n-1}{ }_{2 n-1} C_{n-1} \cdot \frac{1}{2} \rho, \\
\mu 0^{2 n-1} \epsilon_{1} & =(-)^{n}{ }_{2 n-1} C_{n-1} \cdot \frac{1}{2} \rho .
\end{aligned}
$$

The first two of these have already been stated in (31).

(iii) For the other differences, we may regard the table as conpounded of two, in which the values of $\epsilon$ are respectively

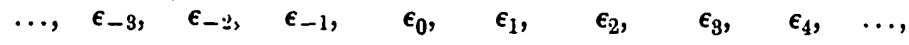

$$
\begin{aligned}
& \ldots,-\frac{1}{2} \rho,+\frac{1}{2} \rho,-\frac{1}{2} \rho,+\frac{1}{2} \rho,-\frac{1}{2} \rho,+\frac{1}{2} \rho,-\frac{1}{2} \rho,+\frac{1}{2} \rho, \ldots, \\
& \ldots, \quad 0, \quad 0, \quad 0, \quad 0, \quad+\rho,-\rho,+\rho,-\rho, \ldots
\end{aligned}
$$

Examination of the derivation of the successive differences in each of the two tables will show that, if $p+m>0$, but $p \leqslant 0$,

$$
\Delta^{m} \epsilon_{p}=(-)^{p+m-1}\left\{{ }_{m} C_{p+m-1}^{\prime}-2^{m-1}\right\} \rho,
$$

where

$$
\begin{aligned}
{ }_{n} C_{s}^{\prime} & \equiv{ }_{n n} C_{0}+{ }_{n} C_{1}+\ldots+{ }_{n} C_{s} \\
& =\text { coeff. } x^{s} \text { in expansion of }(1+x)^{m} /(1-x) .
\end{aligned}
$$

It is clear that ${ }_{m} C_{s}^{\prime} \lesseqgtr 2^{m-1}$, according as $s \lesseqgtr \frac{1}{2}(m-1)$, and therefore $\Delta^{m} \epsilon_{p}$ is of sign $(-)^{p+n}$ or $(-)^{p+n-1}$ according as $p$ is less or greater than $-\frac{1}{2}(m-1)$, being zero if $p=-\frac{1}{2}(m-1)$.

The formula (78) also holds if $p>0$, since in that case ${ }_{m} C_{p+m-1}^{\prime}=2^{m}$, so that $\Delta^{m} \epsilon_{p}=(-)^{p+m-1} 2^{m-1} \rho$, as in (76).

(iv) (a) If $p \leqslant 0$ and $p+m>0$, so that $u_{0}$ and $u_{1}$ occur in ${ }_{m} L_{\theta-p}$ ${ }_{m} E_{\theta-p}$ is positive;

(b) If $p>0,{ }_{m} E_{\theta-p}$ is of sign $(-)^{p+1}$;

(c) If $p+m \leqslant 0,{ }_{m} E_{\theta-p}$ is of $\operatorname{sign}(-)^{p+m}$.

(v) Since $\delta^{2 n+1} \epsilon_{\ddagger}=0$, it follows from (66) and (67) that

$$
{ }_{2 n+1} E_{\theta+n}={ }_{2 n} E_{\theta+n}={ }_{2 n} E_{\theta+n-1} \text {. }
$$




\section{Relative Accuracy of Formula.}

21. We have already found that the limit of tabular error is less for the central-difference formula than for the advancing-difference formula of the same (i.e., the $m$-th) order. These two formulæ correspond to the use of ${ }_{n} L_{\theta-p}$ with different values of $p$, i.e., they correspond to two particular positions of the tabular triangle of a difference of order $m$. We have now to consider the limit of error in the more general case, i.e., for any arbitrary position of the tabular triangle.

We can most conveniently do this by taking a particular position of the triangle, and then seeing how the corresponding value of ${ }_{n} E_{\theta-n}$ is altered by shifting the triangle in one direction or the other.

22. Taking first the central-difference formula :-

(i) If we go up to a difference of order $2 n+1$, this difference will be $\delta^{2 n+1} u_{\frac{1}{2}}$; and the values of $u$ involved will be from $u_{-n}$ to $u_{n+1}$. The limit of tabular error will therefore be $\pm_{2 n+1} E_{\theta+n}$.

(ii) Suppose we only go up to the difference of order $2 n$. Then the values of $u$ involved will be from $u_{-n}$ to $u_{n}$ if $\theta$ lies between 0 and $\frac{7}{2}$, and from $u_{-n+1}$ to $u_{n+1}$ if $\theta$ lies between $\frac{1}{2}$ and 1 ; and the limit of error will be $\pm_{2 n} E_{\theta+n}$ in the one case, and $\pm_{2_{n}} E_{\theta+n-1}$ in the other. But each of these, by (80), is equal to $\pm_{2 n+1} E_{\theta+n}$.

(iii) Hence the limit of tabular error for the central-difference formula up to the term involving a difference of order $2 n$ or $2 n+1$ is $\pm_{2 n+1} E_{\theta+n}$.

23. Next, suppose that we are using any other difference-formula, equivalent to the Lagrange-formula (59); the $u$ 's involved being from $u_{p}$ to $u_{p+m}$, so that the $u$ 's and differences which occur in the formula lie within the tabular triangle of $\Delta^{m} u_{p}$. Then the limit of tabular error is $\pm_{n} E_{\theta-p}$. Let us see how this limit is affected by moving the tabular triangle nearer to the position which it occupies for the central-difference forroula.

(i) For the central-difference formula, the vertex of the tabular triangle lies either within ( $m$ odd) or on the boundary of ( $m$ even) the central horizontal strip bounded by lines through $\theta=0$ and $\theta=1$; and for any other formula, except in the special case mentioned below, it lies outside this strip.

(ii) The exceptional case is that in which $m$ is even, and $\Delta^{\prime \prime \prime} u_{p}$ is either $\delta^{m} u_{1}$, where $\theta$ lies between 0 and $\frac{1}{2}$, or $\delta^{m} u_{0}$, where $\theta$ lies between $\frac{1}{2}$ and 1 . The value of ${ }_{m} E_{\theta-p}$ is then ${ }_{m} E_{\theta+\jmath_{m-1}}$ or ${ }_{n} E_{\theta+3 n}$, while for the centraldifference formula it would be ${ }_{m} E_{\theta+\frac{1}{2} m}$ or ${ }_{m} E_{\theta+\frac{1}{2} n-1}$. But, by (80),

$$
{ }_{m} E_{\theta+3 m}={ }_{m} E_{\theta+1 m-1} \text {. }
$$


Hence, if we go up to a difference of even order $m$, it is immaterial, so far ns the tabular error is concerned, whether this is $\delta^{m} u_{0}$ or $\delta^{m} u_{1} ; \theta$ being between 0 and 1 [cf. $\$ 12$ ].

(iii) For any other case, the vertex of the triangle lies outside the central strip, being on the positive or the negative side of it according as

$$
p>-\frac{1}{2} m+1 \text { or }<-\frac{1}{2} m \text {. }
$$

(iv) First suppose that $p>-\frac{1}{2} m+1$. Then the effect of shifting the triangle backwards one place is to alter the limit of error from $\pm_{m} E_{\theta-p}$ to $\pm_{m} E_{\theta-\eta+1}$. But, by (66) and (67),

$$
{ }_{m} E_{\theta-p}-{ }_{m} E_{\theta-p+1}=\frac{(\theta-p)(\theta-p-1) \ldots(\theta-p-m+1)}{m !} \Delta^{m+1} \epsilon_{p-1} ;
$$

and there are three cases to be considered.

(a) Usually the triangle will include $u_{0}$, so that $p \leqslant 0$. Then $\Delta^{m+1} \epsilon_{p-1}$ will, by $\$ 20$ (iii), be of sign $(-)^{p+m-1}$, and its coefficient in (81) will include $-p+1$ positive, and $p+m-1$ negative, factors. The expression on the right-hand side of (81) will thergfore be positive. Also, by $\$ 20$ (iv) $(a),{ }_{m} E_{\theta-p}$ and ${ }_{n} E_{\theta-p+1}$ are both positive. Hence ${ }_{m} E_{\theta-p+1}$ is less than ${ }_{m} E_{\theta-p}$, so that the shift will reduce the limit of error.

(b) Next, let $p$ be $>1$, so that $u_{0}$ will not be within the triangle, either in its old or in its new position. Then ${ }_{n k} E_{\theta-p}$ and ${ }_{m} E_{\theta-p+1}$ will [see $\$ 20$ (iv) (b)] be of opposite signs. But, if they are expressed in the Lagrange form, the terms in each will all be of the same sign ; and it will be found that each term in $\left|{ }_{m} E_{\theta-p}\right|$ is greater than the corresponding term in $\left|{ }_{m} E_{\theta-p+1}\right|$. Hence* $\left|{ }_{n} E_{\theta-p}\right|>\left|{ }_{m} E_{\theta-p+1}\right|$, and the shift will reduce the limit of error.

(c) Finally, let $p=1$, so that the shift will bring $u_{0}$ within the triangle. Then ${ }_{m} E_{\theta-p}$ and ${ }_{m} E_{\theta-r+1}$ are both positive, and the reasoning of (a) applies.

(d) Hence, in every case, the effect of shifting the tabular triangle one place backwards is to reduce the limit of error.

(v) Similarly, if $p<-\frac{1}{2} m$, so that the vertex of the triangle lies on the negative side of the central strip, we reduce the limit of error by shifting the triangle one place forwards.

* We might also obtain this result by regarding the tabular triangle as fixed, and $\theta$ as altering continuously to $\theta+1$. It is easy to see that, so long as $p>\theta,\left|m E_{\theta-p}\right|$ is always of the same form, and $\left.d\right|_{\ldots} E_{\theta-p} \mid / d \theta$ is negative. 
(vi) It follows that, if our formula is not a central-difference formula (in the general sense of $\S 16$ ), we can reduce the limit of tabular error by replacing the formula by a more central one; in other words, the centraldifference formula gives a smaller limit of tabular error than any other formula of this kind, involving the same number of consecutive values of $u$. This is subject to the exception mentioned in (ii), where the difference of highest order, instead of being the central-difference on one boundary of the central strip, is the corresponding difference on the other boundary; in this case the substitution of the correct central-difference formula does not reduce the limit of error, but it does not increase it.

24. Instead of moving the vertex of the tabular triangle directly from $\Delta^{m} u_{\mathrm{p}}$ to $\Delta^{m} u_{p-1}$, we might move it by two diagonal steps, either first outwards (away from the line of values of $x$ ) and then inwards, or first inwards and then outwards. In the first case, the successive formulæ for $u_{\theta}$ would be ${ }_{m} L_{\theta-1, m_{+1}} L_{\theta-p+1},{ }_{n} L_{\theta-p+1}$, and in the second case they would be ${ }_{n} L_{\theta-p},{ }_{n-1} L_{\theta-p},{ }_{n} L_{\theta-p+1}$. This suggests the comparison of the limits of error for tabular triangles whose vertices are diagonally adjacent; e.g., the comparison of ${ }_{m} E_{\theta-p}$ with ${ }_{m+1} E_{\theta-p+1}$ or with ${ }_{m-1} E_{\theta-p}$.

(i) For movements within the central strip, we have already found [§§ 20 (v) and 22] that ${ }_{2 n} E_{\theta+n-1}={ }_{2 n+1} E_{\theta+n}={ }_{2 n} E_{\theta+n}$. Hence, by starting from one boundary of the strip, moving outwards to the centre, and then inwards to the other boundary, we make no alteration in the limit of error. If we moved first inwards and then outwards, we should first decrease it and then increase it.

(ii) Next, suppose that in the first position the vertex lies outside the central strip, on the positive side of it. Then, by (66) and (67),

$$
\begin{aligned}
& { }_{m} E_{\theta-p}={ }_{m-1} E_{\theta-p}+\frac{(\theta-p)(\theta-p-1) \ldots(\theta-p-m+1)}{m !} \Delta^{m} \epsilon_{p}, \\
& { }_{m} E_{\theta-p}={ }_{m+1} E_{\theta-p+1}-\frac{(\theta-p)(\theta-p-1) \ldots(\theta-p-m)}{(m+1) !} \Delta^{m+1} \epsilon_{p-1} ;
\end{aligned}
$$

and, by $\S 20$ (iii), $\Delta^{m} \epsilon_{p}$ and $\Delta^{m+1} \epsilon_{p-1}$ are of sign $(-)^{p+m-1}$.

(a) If $p \leqslant 1$, so that the first triangle includes $u_{1}$, the term added in (82) is positive, and the term subtracted in (83) is negative; and, by $\S 20$ (iv) (a) and (b), ${ }_{m} E_{\theta-p, n-1} E_{\theta-p}$, and ${ }_{n+1} E_{\theta-p+1}$ are all positive. Hence ${ }_{1 m} E_{\theta-p}$ is greater than each of the other two, so that the limit of error is reduced by moving diagonally towards the central strip, whether inwards or outwards. 
(b) If $p>1$, then ${ }_{m} E_{\theta-p,{ }_{m-1}} E_{\theta-p}$, and the term added in (82), are all of sign $(-)^{p+1}$. Hence in this case also the limit of error is reduced by moving toward the central strip, provided that we move inwards.

(c) This, however, will not necessarily be the case if $p>1$ and we move outwards. Since ${ }_{m} E_{\theta-p}$ and ${ }_{m+1} E_{\theta-p+1}$ are respectively of signs $(-)^{p+1}$ and $(-)^{p}$, we have to compare the values of $(-)^{n+1}{ }_{m} E_{\theta-p}$ and $(-)^{p}{ }_{n+1} E_{\theta-p+1}$. We have

$$
\begin{aligned}
(-)^{m+1}{ }_{m} E_{\theta-p}=\frac{(p-\theta)(p+1-\theta) \ldots(p+m-\theta)}{m !} & \\
& \times\left\{\frac{{ }_{m} C_{0}}{p-\theta}+\frac{{ }_{m} C_{1}}{p+1-\theta}+\ldots+\frac{{ }_{m} C_{m}}{p+m-\theta}\right) \frac{1}{2} \rho, \\
(-)^{\prime \prime}{ }_{m+1} E_{\theta-p+1}=\frac{(p-1-\theta)(p-\theta) \ldots(p+m-\theta)}{(m+1) !} & \left.\times ! \frac{{ }^{m+1} C_{0}}{p-1-\theta}+\frac{{ }^{m+1} C_{1}}{p-\theta}+\ldots+\frac{{ }_{m+1} C_{m+1}}{p+m-\theta}\right) ! \frac{1}{2} \rho ;
\end{aligned}
$$

so that, denoting the expressions in the curled brackets by $S_{n}$ and $S_{m+1}^{\prime}$, we have to compare the values of $(m+1) S_{m}$ and $(p-1-()) S_{m+1}^{\prime}$. Now

Also

$$
\begin{aligned}
& S_{m}=\int_{0}^{1} x^{p-1-\theta}(1+x)^{m} d x, \quad S_{m+1}^{\prime}=\int_{0}^{1} x^{p-2-\theta}(1+x)^{m+1} d x, \\
& (m+1) S_{m}+(p-1-\theta) S_{m+1}^{\prime}=2^{m+1} \text {. }
\end{aligned}
$$

$$
\begin{aligned}
\frac{p-1-\theta}{m+1} S_{m+1}^{\prime}=\frac{1}{m+1} & +(p-1-\theta) \frac{{ }_{m} C_{0}}{p-\theta} \\
& +\frac{p-1-\theta}{2} \frac{{ }_{m} C_{1}}{p+1-\theta}+\ldots+\frac{p-1-\theta}{m+1} \frac{{ }^{m} C_{m}}{p+m-\theta} .
\end{aligned}
$$

This can obviously be greater than $S_{m}$ if $p-1-\theta$ is very great, e.g., if $p-1-\theta>m+1$; but, if $p=2$, so that $p-1-\theta=1-\theta$, the sum of the terms after the first can be made as small as we please by taking $1-\theta$ small enough, so that the sum of all the terms can be made less than $2^{m} /(m+1)$, and therefore less than $S_{m}$. Hence, by moving outwards towards the central strip, the limit of error will be in some cases increased and in other cases decreased.

(iii) We shall, of course, get corresponding results if the vertex of the triangle lies on the negative side of the central strip.

(iv) Hence the limit of error is in all cases reduced by moving the vertex of the tabular triangle diagonally inwards towards the central line; and it is also reduced by moving the vertex diagonally outwards towards the central line, if the triangle in its new position includes both $u_{0}$ and $u_{1}$. 
25. In shifting the tabular triangle towards the centre, we are in effect taking away one of the extreme values of $u$ and replacing it by a value at the other end, nearer the centre. It might be inferred that, in the more general case to which the general Lagrange-formula applies, i.e., the case in which the differences between successive values of $x$ are not all equal, we should reduce the tabular error by replacing one of the values of $u$ by a value nearer the value corresponding to $x$. This, however, is not the case; the tabular error might be increased. Suppose, for instance, that $\theta=\cdot 2$, and that our formula in the first instance involves $u_{0}, u_{1}$, and $u_{2}$. If instead of $u_{0}, u_{1}$, and $u_{2}$ we use $u_{0}, u_{\frac{1}{2}}$, and $u_{1}, \theta$ becomes $\cdot 4$ of the new interval $\frac{1}{2} h$ in $x$; and it will be seen from Table $I(\$ 8)$ that the limit of tabular error is increased from $\pm 580 \rho$ to $\pm 620 \rho$. The residual error, however, is likely to be reduced by the change; and it might even become possible to dispense with 2nd differences, the limit of tabular error being thus reduced to $\pm 500 \rho$.

\section{Corrected Differences.}

26. In comparing the limits of tabular error for different formulæ, we have assumed that the table used for the interpolation is arranged so as to show the tabular differences, i.e., that each quantity in any column (after that of $u$ ) is the exact difference of the two adjoining quantities in the preceding column. There is an alternative system of tabulation which is sometimes adopted ; on this system the values of the differences, like those of the $u$ 's, are "corrected" in the final figure, so that each difference is given within the same limits of error $\pm \frac{1}{2} \rho$ as each $u$. We have to see what effect the use of these corrected differences would have on the limits of error of $u_{\theta}$ as given by the different formulæ.

27. If we obtain $u_{\theta}$ by applying a formula which involves differences to a table which shows corrected differences, the tabular error in the value so obtained will lie between $-R$ and $+R$, where $R$ is found by replacing each difference (and each $u$ ) in the formula by $-\frac{1}{2} \rho$ or $+\frac{1}{2} \rho$ according as the coefficient of the difference (or of the $u$ ) is negative or positive. If, for instance, we use the advancing-difference formula (3), we shall have

$$
R=\left\{1+\frac{\theta}{1 !}+\frac{\theta(1-\theta)}{2 !}+\frac{\theta(1-\theta)(2-\theta)}{3 !}+\ldots\right\} \frac{1}{2} \rho .
$$

Similarly, for the standard central-difference formula (16), $\theta$ being between 0 and $\frac{1}{2}$, we shall have

$$
R=\left\{1+\frac{\theta}{1 !}+\frac{\theta(1-\theta)}{2 !}+\frac{(1+\theta) \theta(1-\theta)}{3 !}+\ldots\right\} \frac{1}{2} \rho .
$$

It is true that, as will be shown in more detail in $\$ 35$ below, the range 
of the possible error in any particular case is less than from $-R$ to $+R$; but it is simpler to take these limits first and to consider later whether, and to what extent, our results need modification. To avoid repetition of the \pm sign, we may call $R$ the limit of error.

It must be observed that formulæ which, on the system of tabular differences, are identical in result $[\$ 14]$ and have the same limit of error, may on the system of corrected differences have different limits of error. The expressions $u_{0}+\theta\left(u_{1}-u_{0}\right)$ and $u_{1}-(1-\theta)\left(u_{1}-u_{0}\right)$, for instance, are identically equal, but $u_{0}+\theta \Delta u_{0}$ and $u_{1}-(1-\theta) \Delta u_{0}$ will have limits of error $(1+\theta) \frac{1}{2} \rho$ and $(2-\theta) \frac{1}{2} \rho$ respectively. The methods used in $\S \S 21-24$ for comparing the limits of error of different formulæ are therefore no longer applicable ; and we have to take into account not only the formulæ of the ordinary kind, constructed as in $\$ 15$, but also the variants, such as Everett's formula, mentioned in $\$ 17$.

28. Considering first the advancing-difference formula, we see that if we have not to go beyond 1st or 2nd differences, we get a worse result by using corrected differences. For 1st differences the limit of error is increased from $\frac{1}{2} \rho$ to $(1+\theta) \frac{1}{2} \rho$. For 2nd differences, instead of

we get

$$
\begin{gathered}
\{1+\theta(1-\theta)\} \frac{1}{2} \rho, \\
\left\{1+\theta+\frac{1}{2} \theta(1-\theta)\right\} \frac{1}{2} \rho .
\end{gathered}
$$

It might be supposed that the same result holds when the series is continued indefinitely. Thus one writer* says: "Il est facile de conclure de ce que précède que l'on doit également préférer les différences tabulaires pour les ordres superieurs, lorsqu'il est nécessaire d'en faire usage." This conclusion, however, can easily be shown to be incorrect. For tabular differences the limit of error is given by (9), and the series inside the brackets is divergent; but the corresponding formula for corrected differences is (84), and the sum of the first $m+1$ terms inside the brackets is

$$
2-\frac{(1-\theta)(2-\theta) \ldots(m-\theta)}{m !},
$$

so that the series is convergent. Hence, whatever the value of $\theta$ may be (between 0 and 1), there is always a number $m$ such that, if we have to go up to differences of order not less than $m$, corrected differences give in better result than tabular differences.

This is also the case with the central-difference formula; the series in (32) is a hypergeometric series, which is divergent, + but the series in brackets in (85) is convergent [see $\S 33$ below].

* F. Lefort, Proc. Roy. Soc., Edinburgb, Vol, vinI (1874-5), p. 610.

† G. Chrystal, Algebra, Pt. II (1st ed.), p. 116, Ex. 2 ; or T. J. I'A. Bromwich, Introduction to the Theory of Infinite Series, p. 35, Ex.3. 
29. Before making any further comparison between the two systems, let us compare the limits of error for the different formulæ of the kind considered in $\$ 15$, on the assumption that corrected differences are used throughout.

(i) Suppose that we have to go up to a difference of order $m$. Compare two formulæ, which are identical as far as a term involving $\Delta^{m-1} u_{p}$ inclusive. Then the difference involved in the remaining term will be $\Delta^{m} u_{p-1}$ in one of the formulæ and $\Delta^{m} u_{p}$ in the other. But the coefficient of this difference will be the same in both formulæ, viz.,

$$
\frac{(\theta-p)(\theta-p-1) \ldots(\theta-p-m+1)}{m !} ;
$$

and therefore the two formulæ will have the same limit of error.

(ii) Next compare two formulæ, which are identical up to a term in $\Delta^{r-1} u_{q}$ (inclusive) and beyond a term in $\Delta^{r+1} u_{t-1}$ (exclusive), but differ in that the intermediate term involves $\Delta^{r} u_{1-1}$ in the one formula and $\Delta^{r} u_{l_{1}}$ in the other. This intermediate term and the next term will in the one formula be

$$
\begin{aligned}
\frac{(\theta-q)(\theta-q-1) \ldots(\theta-q-r+1)}{r !} \Delta^{r} u_{q-1} & \\
& +\frac{(\theta-q+1)(\theta-q) \ldots(\theta-q-r+1)}{(r+1) !} \Delta^{r+1} u_{\eta-1},
\end{aligned}
$$

and in the other

$$
\begin{aligned}
\frac{(\theta-q)(\theta-q-1) \ldots(\theta-q-r+1)}{r !} & \Delta^{r} u_{q} \\
+ & +\frac{(\theta-q)(\theta-q-1) \ldots(\theta-q-r)}{(r+1) !} \Delta^{r+1} u_{\eta-1} ;
\end{aligned}
$$

and the limit of error for the former will be greater or less than for the latter according as $|\theta-q+1|>\langle|\theta-q-r|$, i.e., according as the horizontal line through $x_{q-1}$ or through $x_{\eta_{+r}}$ is the further from that through $x_{\theta}$, i.e., according as $\Delta^{r-1} u_{q}$ lies on the negative or on the positive side of the horizontal line through $x_{\theta}$. Hence we make our limit of error smaller by using that one of the two differences $\Delta^{r} u_{\eta-1}$ and $\Delta^{r} u_{\eta}$ which lies the nearer to this line.

(iii) It follows from (ii) that, if we have to end with a specified difference $\Delta^{m} u_{p}$, we get the best result by keeping as near as possible to the $x_{\theta}$ horizontal line (i.e., by using $u_{0}, \delta u_{2}, \delta^{2} u_{0}, \ldots$, if $0<\theta \leqslant \frac{1}{2}$ or $u_{1}, \delta u_{1}, \delta^{2} u_{1}, \ldots$, if $\frac{1}{2} \leqslant \theta<1$ ) until it becomes necessary to move 
diagonally towards $\lrcorner^{m} u_{p}$. And it follows from successive applications of (i) and (ii) that the central-difference formula (in the strict sense) gives the smallest limit of error.

30. We can now go on to compare the limits of error, on the two sjstems, due to the principal formulæ, viz., the advancing-difference formula (3), the standard central-difference formula (16) (for $0<\theta \leqslant \frac{1}{2}$ ), and the rariants (18) and (19) (for $0<\theta<1$ ), and (20) and (21) (for $0<\theta \leqslant \frac{1}{2}$ ). Using " $\alpha$ " and " $\beta$ " to denote the effects of taking tabular differences and corrected differences respectively, we obtain the following table, the factor $\frac{1}{2} \rho$ being omitted.

TABLE III.

( $\alpha=$ tabular differences. $\quad \beta=$ corrected differences.)

\begin{tabular}{|c|c|c|c|c|c|c|}
\hline \multirow{2}{*}{ Formula. } & \multicolumn{6}{|c|}{ Limit of error $\left(\div \frac{1}{2} \nu\right)$ due to using } \\
\hline & $u$ & 1st diff. & 2nd diff. & 3rd diff. & 4th diff. & 5 th diff. \\
\hline$(3 a)$ & 1 & +0 & $+\theta(1-\theta)$ & $+\theta(1-\theta)(2-\theta)$ & $+\frac{7}{12} \theta(1-\theta)(2-\theta)(3-\theta)$ & $+\frac{1}{4} \theta(1-\theta)(2-\theta)(3-\theta)(4-\theta)$ \\
\hline$(3 \beta)$ & 1 & $+\stackrel{e}{1 !}$ & $+\frac{\theta(1-\theta)}{2 !}$ & $+\frac{\theta(1-\theta)(2-\theta)}{3 !}$ & $+\frac{\theta(1-\theta)(2-\theta)(3-\theta)}{4 !}$ & $+\frac{\theta(1-\theta)(2-\theta)(3-\theta)(4-\theta)}{5 !}$ \\
\hline$(16 a)$ & 1 & +0 & $+\frac{\theta(1-\theta)}{1 ! 1 !}$ & +0 & $+\frac{(1+\theta) \theta(1-\theta)(2-\theta)}{2 ! 2 !}$ & +0 \\
\hline$(16 \beta)$ & 1 & $+\frac{\theta}{1 !}$ & $+\frac{\theta(1-\theta)}{2 !}$ & $+\frac{(1+\theta) \theta(1-\theta)}{3 !}$ & $+\frac{(1+\theta) H(1-\theta)(2-\theta)}{4 !}$ & $+\frac{(2+\theta)(1+\theta) \theta(1-\theta)(2-\theta)}{5 !}$ \\
\hline$(18 \alpha)$ & & 1 & & $+\frac{\theta(1-\theta)}{1 ! 1 !}$ & & $+\frac{(1+\theta) \theta(1-\theta)(2-\theta)}{2 ! 2 !}$ \\
\hline$(18 \beta)$ & & 1 & & $+\frac{\theta(1-\theta)}{2 !}$ & & $+\frac{(1+\theta) \theta(1-\theta)(2-\theta)}{4 !}$ \\
\hline$(19 \alpha)$ & 1 & +0 & $+\frac{\theta(1-H)}{1 ! 1 !}$ & +0 & $+\frac{(1+\theta) \theta(1-\theta)(2-\theta)}{2 ! 2 !}$ & +0 \\
\hline$(19 \beta)$ & 1 & $+\left|\frac{1}{2}-\theta\right|$ & $+\frac{\theta(1-\theta)}{2 !}$ & $+\left|\frac{1}{2}-\theta\right| \frac{\theta(1-\theta)}{3 !}$ & $+\frac{(1+r) \theta(1-\theta)(2-\theta)}{4 !}$ & $+\left|\frac{1}{2}-\theta\right| \frac{(1+H) \theta(1-\theta)(2-\theta)}{5 !}$ \\
\hline$(20 a)$ & 1 & & $+\frac{\theta(1-\theta)}{1 ! 1 !}$ & & $+\frac{(1+\theta) \theta(1-\theta)(2-\theta)}{2 ! 2 !}$ & \\
\hline$(20 \beta)$ & 1 & & $+\frac{\theta}{1 !}$ & & $+\frac{(1+\theta) \theta(1-\theta)}{3 !}$ & \\
\hline$(21 a)$ & 1 & $+\frac{\theta}{1 !}$ & $-\frac{\theta^{2}}{1 ! 1 !}$ & $+\frac{(1+\theta) \theta(1-\theta)}{1 ! 2 !}$ & $-\frac{(1+\theta) \theta^{2}(1-\theta)}{2 ! 2 !}$ & $+\frac{(2+\theta)(1+\theta) \theta(1-\theta)(2-\theta)}{2 ! 3 !}$ \\
\hline$(21 \beta)$ & 1 & $+\frac{\theta}{1 !}$ & $+\frac{\theta^{2}}{2 !}$ & $+\frac{(1+\theta) \theta(1-\theta)}{3 !}$ & $+\frac{(1+\theta) \theta^{2}(1-\theta)}{4 !}$ & $+\frac{(2+\theta)(1+\theta) \theta(1-\theta)(2-\theta)}{5 !}$ \\
\hline
\end{tabular}


This table shows that the effect of using corrected differences is as follows :-

(i) The Everett formula (18), in which the terms are taken in pairs and recast, is the only one in which the terms of the limit of error are decreased throughout.

(ii) For the other formula in which certain terms are taken in pairs, viz. (20), the term due to 1 st and 2 nd differences is increased, while the other terms are decreased.

(iii) For the advancing-difference formula (3), a 1st difference term is introduced, but all the other terms are decreased.

(iv) For (16) and (19) the terms due to differences of even order are decreased (equally in the two formulæ), but new terms due to differences of odd order are introduced. In each case, however, the sum of the terms due to differences of order $2 n$ and $2 n+1$ is (except for $n=0$ ) less in the $\beta$ form than in the $\alpha$ form.

(v) For (21) the terms due to differences of odd order (after the first) are decreased, but those due to differences of even order are increased (algebraically). But the sum of the terms due to differences of order $2 n+1$ and $2 n+2$ is (except for $n=0$ ) less in the $\beta$ form than in the $\alpha$ form.

31. In the case of (3), (16), and (19), the primary disadvantage of using corrected differences is the introduction of a term due to the 1st difference term of the formula. We can avoid this by using tabular 1st differences; and, if we do this, we tind that all three formulæ give better results with corrected differences than with tabular differences. This modified system, under which 1 st differences are tabular and the remainder are corrected, may be denoted by " $\gamma$."

Further, we see that in (18) this result is indirectly achieved by resolving the 1 st difference $\delta u_{3}$ into its components $u_{1}-u_{0}$; and that $a$ similar process is adopted for other differences of odd order. If in (16) or (19) we replace $\delta u_{\frac{1}{2}}, \delta^{3} u_{u_{3}}, \delta^{5} u_{3}, \ldots$ by $u_{1}-u_{0}, \delta^{2} u_{1}-\delta^{2} u_{0}, \delta^{4} u_{1}-\delta^{4} u_{0}, \ldots$ we get (18). Thus we get a new system " $\delta$," under which the differences of even order alone are corrected, while the differences of odd order may be described as "semi-corrected," being the exact differences of the corrected differences of the next lower order. On this system we find that (16) and (19) are improved by taking corrected differences. 
For (3) it will be found that (3ס) is not so good as $(3 \gamma)$. The terms in (3) which involve $\Delta^{n-1} u_{0}$ and $\Delta^{n} u_{0}(n$ being $>2$ ) are respectively

$$
(-)^{n} n f(\theta) \Delta^{n-1} u_{0} \text { and }(-)^{n+1}(n-1-\theta) f(\theta) \Delta^{n} u_{0} \text {, }
$$

where

$$
f(\theta) \equiv \theta(1-\theta)(2-\theta) \ldots(n-2-\theta) / n ! .
$$

The first of these terms, if $\Delta^{n-1} u_{0}$ is corrected, produces in the limit of error a term $n f(\theta) \frac{1}{2} \rho$; and the second, if $\Delta^{n} u_{0}$ is corrected, produces a term $(n-1-\theta) f(\theta) \frac{1}{2} \rho$. If, however, $\Delta^{n} u_{0}$ is the difference of the corrected values of $\Delta^{n-1} u_{1}$ and $\Delta^{n-1} u_{0}$, the two terms of (3) are [ $\$ 17$ (i)] together equal to

$$
(-)^{n} f(\theta)\left\{(\theta-n+1) \Delta^{n-1} u_{1}-(\theta-2 n+1) \Delta^{n-1} u_{0}\right\} \text {; }
$$

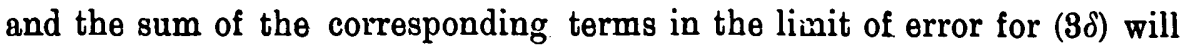
be

$$
f(\theta)\{(n-1-\theta)+(2 n-1-\theta)\} \frac{1}{2} \rho=n f(\theta) \frac{1}{2} \rho+2(n-1-\theta) f(\theta) \frac{1}{2} \rho .
$$

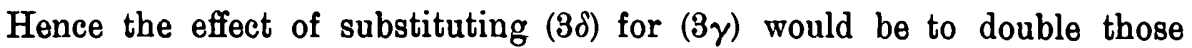
terms in the limit of error which are due to differences of odd order. On the other hand, the terms for $(3 \delta)$ are still individually less than for $(3 \alpha)$.

The formulæ (20) and (21) differ from (18) and (19) in that they are central to $u_{0}$ instead of to $u_{3}$, so that the terms which go in pairs are of odd and even order, not of even and odd. In order to keep the limit of error as low as possible, we must obviously use tabular 1st differences for both formulæ, and tabular 2nd difference for the third term in (21). For the remaining terms we have a choice of three systems : (i) all differences above the 2nd corrected ; (ii) differences of the $3 \mathrm{rd}$, 5 th, ... orders semicorrected and those of the $4 \mathrm{th}, 6 \mathrm{th}, \ldots$ orders corrected ; (iii) differences of the $3 \mathrm{rd}, 5 \mathrm{th}, \ldots$ orders corrected and those of the $4 \mathrm{th}, 6 \mathrm{th}, \ldots$ orders semi-corrected ; these systems may be denoted by " $\gamma$," " $\delta^{\prime}$," and "e." As regards (20), it is clear that $(20 \epsilon)$ and $\left(20 \gamma^{\prime}\right)$ are identical with $(20 \gamma)$, while $\left(20 \delta^{\prime}\right)$ is identical with $(20 \delta)$; all five systems give $\{1+\theta(1-\theta)\} \frac{1}{2} \rho$ as due to the first three terms, and for $(20 \gamma),\left(20 \gamma^{\prime}\right)$, and $(20 \epsilon)$ the remaining terms are the same as for $(20 \beta)$, while for $(20 \delta)$ and $\left(20 \delta^{\prime}\right)$ they are

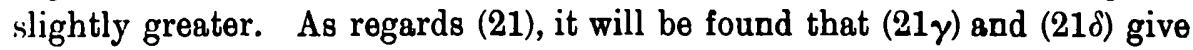
the same result as $(21 \beta)$, and $\left(21 \gamma^{\prime}\right)$ and $\left(21 \delta^{\prime}\right)$ give the first three terms as in $(21 \alpha)$ and the remainder as in $(21 \beta)$; but $(21 \epsilon)$ differs from $\left(21 \gamma^{\prime}\right)$ and $\left(21 \delta^{\prime}\right)$ in that it gets rid of the terms which, in $(21 \beta)$, are due to differences of the 4th, 6th, ... order. Up to differences of an even order, $(20 \epsilon)$ and $(21 \epsilon)$ are of course identical.

The more important of these results are shown in the following table. It should be specially remembered that for $\left(21 \delta^{\prime}\right)$ we start with having all differences of even order corrected, but that we use the tabular value of $\delta^{2} u_{0}$ in the formula, although we use the corrected values of $\delta^{2} u_{-1}$ and of $\delta^{2} u_{1}$ for determining $\mu \delta^{9} u_{0}$. 


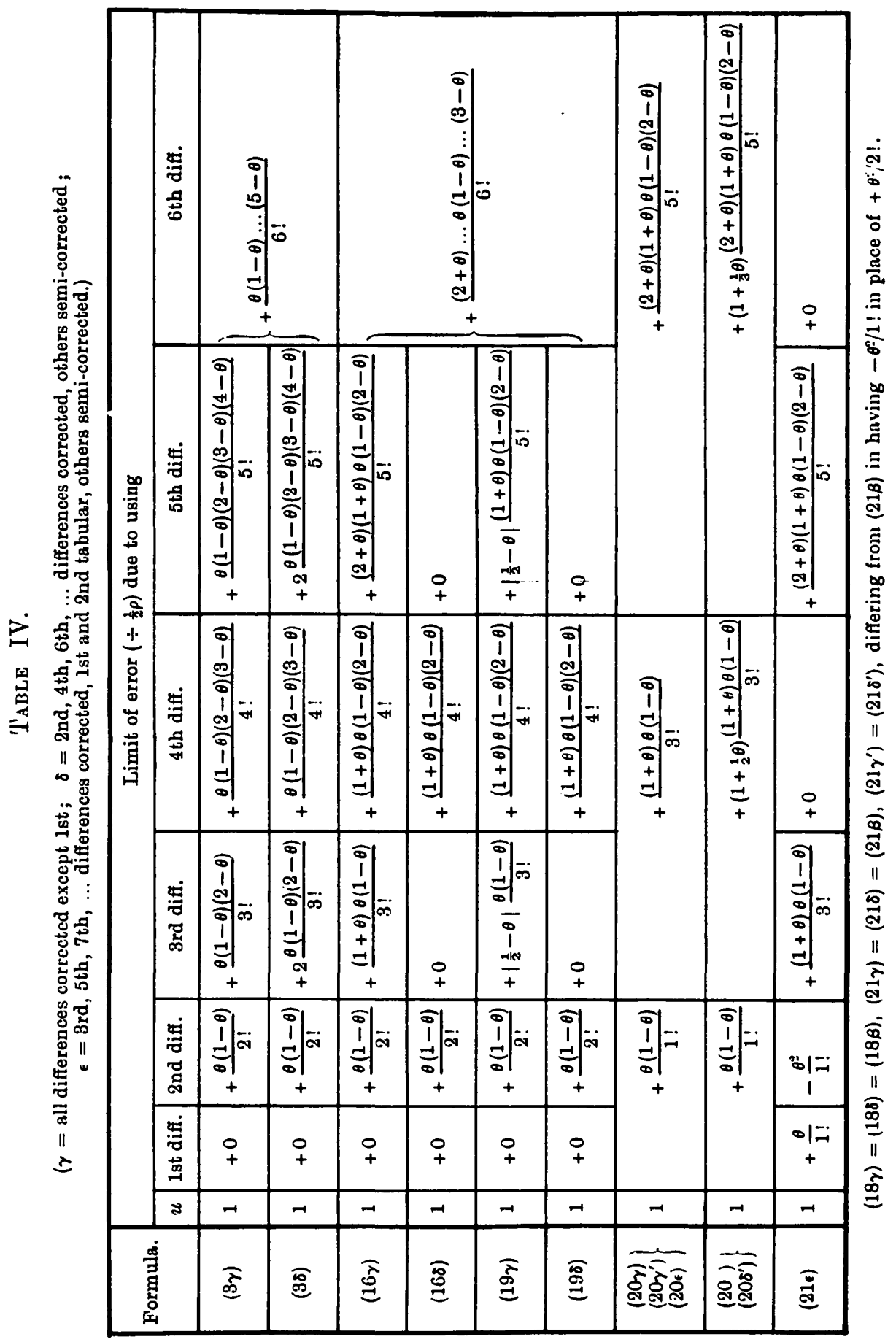


32. Without going exhaustively into the various possible formulæ, we can check the more important of these results by a method which is an extension of that applied in $\S 31$ to the comparison of $(3 \gamma)$ and $(3 \delta)$. Suppose that we have a formula which ends at $\Delta^{m} u_{-p}$ with a corrected difference; let us see how the subsequent differences should be treated.

(i) Let the term in $\Delta^{m} u_{-p}$ be $f_{1}(\theta) \Delta^{m} u_{-p}$, so that it introduces into the limit of error a term $\left|f_{1}(\theta)\right| \frac{1}{2} \rho$; and let the coefficient of the next difference, which is either $\Delta^{m+1} u_{-p}$ or $\Delta^{m+1} u_{-,-1}$, be $f_{2}(\theta)$. Then

(a) If this difference also is corrected, it will introduce a term

$$
\left|f_{2}(\theta)\right| \frac{1}{2} \rho \text {. }
$$

$(\beta)$ If it is semi-corrected, we have to combine the two terms of the formula ; and they will be found to give rise to

or to

$$
\left\{f_{1}(\theta)-f_{2}(\theta)\right\} \Delta^{n z} u_{-p}+f_{2}(\theta) \Delta^{m} u_{-p+1},
$$

according as the step is forwards or backwards. In the former case the total of the corresponding terms of the limit of error is

$$
\left\{\left|f_{1}(\theta)-f_{2}(\theta)\right|+\left|f_{2}(\theta)\right|\right\} \frac{1}{2} \rho,
$$

and in the latter case it is

$$
\left\{\left|f_{1}(\theta)+f_{2}(\theta)\right|+\left|f_{2}(\theta)\right|\right\} \frac{1}{2} \rho .
$$

Also (if $0<\theta<1$, and if we start with $u_{0}$ or $u_{1}$ and $\Delta u_{0}$ )

$$
\left|f_{1}(\theta)\right|>\left|f_{2}(\theta)\right| \text {. }
$$

Hence the effect of using the semi-corrected $\Delta^{m+1} u$ will be that the term of the limit of error, due to this term of the formula, will be either $2\left|f_{2}(\theta)\right| \frac{1}{2} \rho$ or 0 ; and it will be 0 if $f_{1}(\theta)$ and $f_{2}(\theta)$ are of the same sign when we are going forwards, or of different sign when we are going backwards. But

$$
f_{2}(\theta) / f_{1}(\theta)=(\theta+p) /(m+1) \text { or }(\theta+p-m) /(m+1),
$$

according as the step to $\Delta^{m} u_{-p}$ brought in $u_{-p}$ or $u_{-p+m}$; i.e., $f_{1}(\theta)$ and $f_{2}(\theta)$ are of the same sign or of opposite sign according as the step to $\Delta^{m} u_{-p}$ was backwards or forwards.

(ii) Hence, if the difference of order $m$ is corrected, and if we have to take in a difference of order $m+1$, the best result is obtained by moving to it in the opposite direction to that in which we last moved, and by expressing the difference of order $m+1$ in terms of (corrected) differences of order $m$. 
(iii) Suppose therefore that the successive differences occurring in the formula are $\Delta^{m-1} u_{-p}, \Delta^{m} u_{-p}$, and $\Delta^{m+1} u_{-p-1}$, the last two being respectively corrected and semi-corrected; and consider the effect of taking in another term. The difference introduced will be either $\lrcorner^{m+2} u_{-p-1}$ or $\Delta^{n+2} u_{-p-2}$, and its coefficient will be

$$
f_{1}(\theta)(\theta+p-m)(\theta+p+1) /\{(m+1)(m+2)\} .
$$

(a) If this difference is corrected or semi-corrected, the term introduced into the limit of error will be

Call this $\lambda$.

$$
\left|f_{1}(\theta)\right|(m-p-\theta)(\theta+p+1) /\left\{( m + 1 ) \left(m+2 ; \cdot \frac{1}{2} \rho .\right.\right.
$$

( $\beta$ ) If the difference is based on the differences of order $m$, it will be found that the total portion of the limit of error, due to the terms of the formula which involve differences of orders $m, m+1$, and $m+2$, will be $\left|f_{1}(\theta)\right| \frac{1}{2} \rho+2 \lambda$, whichever of the two differences of order $m+2$ we take.

We should obtain a similar result by using $\Delta^{m} u_{-p_{-1}}$ instead of $\lrcorner^{n} u_{-r}$.

$(\gamma)$ By using the corrected difference we can [see (i), $(\beta)]$ prevent the next term from increasing the limit of error.

(iv) Hence the best result is obtained by taking the differences alternately corrected and semi-corrected ; i.e., by correcting alternate columns of differences, the intermediate columns being found by differencing these.

(v) The fact that after going forwards from $\Delta^{m-1} u_{-i}$, to $J^{m} u_{-m}$, and then backwards to $\Delta^{m+1} u_{-p-1}$, we may again go backwards to $\Delta^{m+2} u_{-1,-2}$, does not conflict with the rule that we should go alternately backwards and forwards; for the next step should be to $\Delta^{m+3} u_{-p-2}$. Each pair of steps is from one semi-corrected difference to the next in the same horizontal line, and it is immaterial whether the first step of any pair is backwards or forwards.

33. It has already been mentioned [ $\$ 28]$ that the series which gives the limit of error for $(3 \beta)$ is convergent; and it follows from $\$ \$ 29$ and 32 that the corresponding series for the other formulæ we are considering are also convergent. The accuracy of any particular formula can therefore be roughly measured, for purposes of comparison, by the limit of the sum of the series which determines the limit of error. - This may be called the "maximum limit of error." It is assumed throughout that $0<\theta<1$, and that the tabular triangle of the formula always includes the interval $(0,1)$. 
(i) For $(3 \beta)$ the series (omitting the factor $\frac{1}{2} \rho$ ) is

$$
1+\theta+\frac{\theta(1-\theta)}{2 !}+\ldots+\frac{\theta(1-\theta) \ldots(m-1-\theta)}{m !}+\ldots,
$$

the sum of $m+1$ terms of which is

$$
2-\frac{(1-\theta)(2-\theta) \ldots(m-\theta)}{m !} .
$$

The maximum limit of error is therefore $\rho$.

(ii) The series for ( $3 \gamma)$ only differs from the above in the omission of the second term; the maximum limit of error is therefore $\left(1-\frac{1}{2} \theta\right) \rho$.

(iii) The series for ( $(3 \delta)$ is obtained from that for $(3 \gamma)$ by doubling the alternate terms. Remembering that

$$
2^{\theta}=1+\theta+\frac{\theta(\theta-1)}{2 !}+\ldots+\frac{\theta(\theta-1) \ldots(\theta-m+1)}{m !}+\ldots,
$$

it will be found that the maximum limit of error is $\left(1-\theta+1 / 2^{2-\theta}\right) \rho$.

(iv) For $(18 \beta),(18 \gamma)$, and $(18 \delta)$, and also for $(16 \delta)$ and $(19 \delta)$, we have a series

$$
1+\frac{\theta(1-\theta)}{2 !}+\frac{(1+\theta) \theta(1-\theta)(2-\theta)}{4 !}+\ldots .
$$

Writing $\theta \equiv \frac{1}{2}+\psi$, this becomes

But*

$$
1+\frac{\left(\frac{1}{2}\right)^{2}-\psi^{2}}{2 !}+\frac{\left\{\left(\frac{1}{2}\right)^{2}-\psi^{2}\right\}\left\{\left(\frac{3}{2}\right)^{2}-\psi^{2}\right\}}{4 !}+\ldots
$$

$$
\frac{\cos 2 \psi \alpha}{\cos \alpha}=1+\frac{\left(\frac{1}{2}\right)^{2}-\psi^{2}}{2 !}(2 \sin \alpha)^{2}+\frac{\left\{\left(\frac{1}{2}\right)^{2}-\psi^{2}\right\}\left\{\left(\frac{3}{2}\right)^{2}-\psi^{2}\right\}}{4 !}(2 \sin \alpha)^{4}+\ldots
$$

If, therefore, we take $\sin \alpha=\frac{1}{2}, a=\frac{1}{6} \pi$, we find that

$$
1+\frac{\theta(1-\theta)}{2 !}+\frac{(1+\theta) \theta(1-\theta)(2-\theta)}{4 !}+\ldots=\frac{2}{\sqrt{ } 3} \cos \frac{1}{3}\left(\theta-\frac{1}{2}\right) \pi ;
$$

so that the maximum limit of error for any of these formulæ is

$$
\frac{1}{\sqrt{ } 3} \cos \frac{1}{3}\left(\theta-\frac{1}{2}\right) \pi . \rho \text {. }
$$

The limit for $(19 \beta)$ or $(19 \gamma)$ need not be considered, since we should generally use (19ס) in preference.

* E. W. Hobson, Plane Trigonometry, p. 265, formula (7). 
1911.] The aCCURACY OF INTFRPOLATION BY FINITE DIFFERENCES.

(iv) The above series forms part of $(16 \beta)$. The remainder of $(16 \beta)$ is a series

$$
\frac{\theta}{1 !}+\frac{\theta\left(1^{2}-\theta^{2}\right)}{3 !}+\frac{\theta\left(1^{2}-\theta^{2}\right)\left(2^{2}-\theta^{2}\right)}{5 !}+\ldots
$$

which also occurs in $(20 \beta)$ and forms part of $(21 \beta)$ and $(21 \epsilon)$. But*

$\frac{\sin 2 \theta \alpha}{\cos \alpha}=\theta .2 \sin \alpha+\frac{\theta\left(1^{2}-\theta^{2}\right)}{3 !}(2 \sin \alpha)^{3}+\frac{\theta\left(1^{2}-\theta^{2}\right)\left(2^{2}-\theta^{2}\right)}{5 !}(2 \sin \alpha)^{5}+\ldots ;$

and therefore

$$
\frac{\theta}{1 !}+\frac{\theta\left(1^{2}-\theta^{2}\right)}{3 !}+\frac{\theta\left(1^{2}-\theta^{2}\right)\left(2^{2}-\theta^{2}\right)}{5 !}+\ldots=\frac{2}{\sqrt{ } 3} \sin \frac{1}{33} \theta \pi .
$$

The maximum limit of error for $(16 \beta)$ is therefore

$$
\frac{1}{\sqrt{ } 3}\left\{\cos \frac{1}{3}\left(\theta-\frac{1}{2}\right) \pi+\sin \frac{1}{3} \theta \pi\right\} \rho=\cos \frac{1}{: 3}(1-\theta) \pi . \rho .
$$

For $(16 \gamma)$ it is $\quad\left\{\cos \frac{1}{3}(1-\theta) \pi-\frac{1}{2} \theta ! \rho\right.$;

for $(20 \beta)$ it is $\quad\left(\frac{1}{2}+\frac{1}{\sqrt{ } 3} \sin \frac{1}{! 3} \theta_{\pi}\right) \rho$;

and for $(20 \gamma),\left(20 \gamma^{\prime}\right),(20 \epsilon)$, and $(21 \epsilon)$ it is

$$
\left\{\frac{1}{2}\left(1-\theta^{2}\right)+\frac{1}{\sqrt{ } 3} \sin \frac{1}{35} \theta_{\pi} ; \rho .\right.
$$

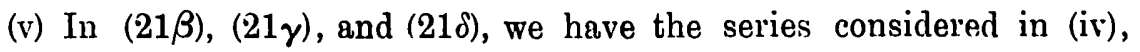
and also a series

$$
1+\frac{\theta^{2}}{2 !}+\frac{\theta^{2}\left(1^{2}-\theta^{2}\right)}{4 !}+\frac{\theta^{2}\left(1^{2}-\theta^{2}\right)\left(2^{2}-\theta^{2}\right)}{6 !}+\ldots
$$

which may similarly be shown to be equal to $2-\cos { }_{:}^{1} \theta \pi$. Hence the maximum limit for any of these three formulæ is

$$
\left(1-\frac{1}{2} \cos \frac{1}{3} \theta \pi+\frac{1}{\sqrt{ } 3} \sin \frac{1}{3} \theta \pi\right) \rho ;
$$

and for $\left(21 \gamma^{\prime}\right)$ and $\left(21 \delta^{\prime}\right)$ it is

$$
\left(1-\frac{3}{4} \theta^{2}-\frac{1}{2} \cos \frac{1}{3} \theta \pi+\frac{1}{\sqrt{ } 3} \sin \frac{1}{3} \theta \pi\right) \rho .
$$

STiR. 2. VOL. 10 . No. 110 .

- Ibid., formula (8). 
For $(20 i)$ and $\left(20 \delta^{\prime}\right)$ the maximum limit will be found to be

$$
\left.\left(\frac{3}{2}-\theta^{2}-\cos \frac{1}{3} \theta \pi+\frac{1}{\sqrt{ } 3} \sin \frac{1}{3} \theta \pi\right) \rho=\frac{1}{3} \frac{3}{2}-\theta^{2}-\frac{2}{\sqrt{ } 3} \sin \frac{1}{3}(1-\theta) \pi\right\}_{j} \rho .
$$

The following table gives the values of the various maximum limits mentioned above, for $\theta=\cdot 1, \cdot 2, \ldots, \cdot 9$. The first portion of the table relates to the systems that might be used with advancing differences, the second portion to central-difference systems that give a low limit of error, and the third to central-difference systems that are inconvenient as giving too high a limit of error, at any rate in the further portion of the interval.

TABLE V.

\begin{tabular}{|c|c|c|c|c|c|c|c|c|c|}
\hline \multirow{2}{*}{$\begin{array}{l}\text { Formula } \\
\text { and } \\
\text { system. }\end{array}$} & \multicolumn{9}{|c|}{ Maximum limit of error $(\div \rho)$ for $\theta=$} \\
\hline & $\cdot 1$ & $\cdot 2$ & $\cdot 3$ & $\cdot 4$ & $\cdot 5$ & $\cdot 6$ & $\cdot 7$ & $\cdot 8$ & $\cdot 9$ \\
\hline$(3 \beta)$ & 1.000 & $1 \cdot 000$ & $1 \cdot 000$ & $1 \cdot 000$ & 1.000 & $1 \cdot 000$ & $1 \cdot 000$ & 1.000 & $1 \cdot 000$ \\
\hline$(3 \gamma)$ & $\cdot 950$ & .900 & $\cdot 850$ & $\cdot 800$ & $\cdot 750$ & $\cdot 700$ & $\cdot 650$ & .600 & $\cdot 550$ \\
\hline$(3 \delta)$ & $1 \cdot 168$ & $1 \cdot 088$ & $1 \cdot 008$ & $\cdot 930$ & $\cdot 854$ & $\cdot 779$ & $\cdot 707$ & $\cdot 630$ & $\cdot 507$ \\
\hline $\begin{array}{c}(16 \delta),(18 \beta), \\
(18 \gamma),(18 \delta), \\
(19 \delta)\end{array}$ & $\cdot 528$ & $\cdot 550$ & $\cdot 565$ & $\cdot 575$ & .578 & $\cdot 575$ & $\cdot 565$ & $\cdot 550$ & $\cdot 528$ \\
\hline$(16 \gamma)$ & $\cdot 538$ & $\cdot 570$ & $\cdot 594$ & 610 & $\cdot 617$ & $\cdot 614$ & $\cdot 602$ & $\cdot 579$ & $\cdot 545$ \\
\hline $\begin{array}{l}(20 \gamma),\left(20 \gamma^{\prime}\right) \\
(20 \epsilon),(21 \epsilon)\end{array}$ & $\cdot 556$ & 601 & $\cdot 634$ & $\cdot 655$ & $\cdot 664$ & $\cdot 660$ & $\cdot 642$ & $\cdot 610$ & $\cdot 563$ \\
\hline$\left(21 \gamma^{\prime}\right)\left(21 \delta^{\prime}\right)$ & $\cdot 556$ & .601 & $\cdot 636$ & $\cdot 659$ & 669 & $\cdot 665$ & $\cdot 648$ & $\cdot 615$ & $\cdot 566$ \\
\hline$(20 \delta), \quad\left(20 \delta^{\prime}\right)$ & .556 & $\cdot 602$ & $\cdot 638$ & $\cdot 662$ & $\cdot 673$ & $\cdot 671$ & $\cdot 654$ & $\cdot 620$ & $\cdot 570$ \\
\hline (20B) & .561 & $\cdot 621$ & $\cdot 679$ & $\cdot 735$ & $\cdot 789$ & .840 & .887 & .930 & .968 \\
\hline $\begin{array}{c}(21 \beta),(21 \gamma) \\
(21 \delta)\end{array}$ & ·564 & $\cdot 631$ & $\cdot 703$ & $\cdot 779$ & $\cdot 856$ & $\cdot 935$ & 1.015 & 1.095 & 1.174 \\
\hline$(16 B)$ & $\cdot 588$ & $\cdot 670$ & $\cdot 744$ & $\cdot 810$ & 867 & $\cdot 914$ & $\cdot 952$ & $\cdot 979$ & $\cdot 995$ \\
\hline
\end{tabular}

34. We are now in a position to draw general conclusions as to the applicability of corrected differences to the various formulæ.

(A) For sub-tabulation (construction of tables with smaller intervals) the central-difference formula in Everett's form is still the best, and is improved by using corrected differences. The improvement, however, is not very striking in ordinary cases, since the limit of error with tabular 
differences is itself small, even when an appreciable number of terms is taken. This is shown by the following table.

\section{$\mathrm{T}_{\mathrm{ABLE}} \mathrm{VI}$.}

\begin{tabular}{|c|c|c|c|c|c|c|c|c|c|c|}
\hline \multirow{2}{*}{$\begin{array}{l}\text { Differences } \\
\text { represented } \\
\text { by last pair } \\
\text { of terms. }\end{array}$} & \multirow{2}{*}{$\begin{array}{c}\text { System } \\
\text { of } \\
\text { differences. }\end{array}$} & \multicolumn{9}{|c|}{$\begin{array}{l}\text { Limit of error }(\div \rho) \text { due to using Everett's } \\
\text { formula, for } \theta=\end{array}$} \\
\hline & & $\cdot 1$ & $\cdot 2$ & 3 & $\cdot 4$ & $\cdot 5$ & 6 & $\cdot 7$ & $\cdot 8$ & $\cdot 9$ \\
\hline 1st & $\left\{\begin{array}{l}\text { Tabular } \ldots . . \\
\text { Corrected... }\end{array}\right.$ & $\begin{array}{r}.500 \\
.500\end{array}$ & $\begin{array}{l}.500 \\
.500\end{array}$ & .500 & $\begin{array}{r}\cdot 500 \\
\cdot 500\end{array}$ & $\begin{array}{l}\cdot 500 \\
.500\end{array}$ & $\begin{array}{l}\cdot 500 \\
.500\end{array}$ & $\cdot 500$ & .500 & .500 \\
\hline 2nd and 3rd & $\left\{\begin{array}{l}\text { Tabular } \ldots \\
\text { Corrected } \ldots\end{array}\right.$ & $\begin{array}{l}\cdot 515 \\
.523\end{array}$ & $\begin{array}{l}580 \\
.540\end{array}$ & .605 & $\cdot 620$ & $\cdot 625$ & $\cdot 620$ & $\cdot 605$ & $\cdot 580$ & $\begin{array}{l}\cdot 545 \\
.523\end{array}$ \\
\hline 4 th and 5 th & $\left\{\begin{array}{l}\text { Tabular .... } \\
\text { Corrected... }\end{array}\right.$ & $\begin{array}{l}\cdot 569 \\
\cdot 527\end{array}$ & $\begin{array}{l}.624 \\
.548\end{array}$ & $\begin{array}{l}\cdot 664 \\
\cdot 563\end{array}$ & $\begin{array}{l}\cdot 688 \\
\cdot 572\end{array}$ & $\begin{array}{l}\cdot 696 \\
\cdot 575\end{array}$ & $\begin{array}{l}\cdot 688 \\
\cdot 572\end{array}$ & $\begin{array}{l}.664 \\
.563\end{array}$ & $\begin{array}{l}\cdot 624 \\
\cdot 548\end{array}$ & $\begin{array}{l}.569 \\
.527\end{array}$ \\
\hline fith and 7 th & $\left\{\begin{array}{l}\text { Tabular ... } \\
\text { Corrected... }\end{array}\right.$ & $\begin{array}{l}.585 \\
.528\end{array}$ & $\begin{array}{l}\cdot 653 \\
\cdot 549\end{array}$ & $\begin{array}{l}.704 \\
.565\end{array}$ & $\begin{array}{l}.734 \\
.574\end{array}$ & $\begin{array}{l}.745 \\
.577\end{array}$ & $\begin{array}{l}.734 \\
.574\end{array}$ & $\begin{array}{l}\cdot 704 \\
.565\end{array}$ & $\begin{array}{l}\cdot 653 \\
\cdot 549\end{array}$ & $\begin{array}{l}\cdot 585 \\
\cdot 528\end{array}$ \\
\hline 8 th and 9 th & $\left\{\begin{array}{l}\text { Tabular ... } \\
\text { Corrected... }\end{array}\right.$ & $\begin{array}{l}.597 \\
.528\end{array}$ & $\begin{array}{l}\cdot 676 \\
\cdot 549\end{array}$ & $\begin{array}{l}\cdot 734 \\
\cdot 565\end{array}$ & $\begin{array}{l}.770 \\
.575\end{array}$ & $\begin{array}{l}.792 \\
.578\end{array}$ & $\begin{array}{l}.770 \\
.575\end{array}$ & $\begin{array}{l}\cdot 734 \\
.565\end{array}$ & $\begin{array}{l}\cdot 675 \\
\cdot 549\end{array}$ & $\begin{array}{l}.597 \\
.528\end{array}$ \\
\hline
\end{tabular}

Since differences of even order only are used in the formuln, it is immaterial whether the differences of odd order are corrected or not.

(B) For isolated interpolations the advancing-difference (or recedingdifference) formula is so much improved by the use of corrected differences as to be comparable, so far as the limit of error is concerned, with the central-difference formulæ; and it may in certain cases be found more convenient for use. The following rules may he laid down.

(i) Whatever formula is adopted, the tabular 1st difference must he used, not the corrected 1st difference.

(ii) If we have only to go to 2nd differences, it does not rery much matter whether we use the tabuliur or the corrected 2nd difference. Br a proper use of the corrected difference we can reduce the limit of error from $\left\{1+\theta(1-\theta) ; \frac{1}{2} \rho\right.$ to $\left\{1+\frac{1}{2} \theta(1-\theta)\right\} \frac{1}{2} \rho$; but the difference between these is at most (viz., for $\theta=5$ ) the difference between $\cdot 6250 \rho$ and $5625 \rho$. If we do use the corrected difference, we see from Talsles III and IV that formulæ (20) and (21) are not appropriate ; we can use either

or

(3) $u_{\theta}=u_{0}+\theta \delta u_{3}+\frac{1}{2} \theta(\theta-1) \tilde{\delta}^{2} u_{1}$,

(16) $u_{\theta}=u_{0}+\theta \delta u_{3}+\frac{1}{2} \theta(\theta-1) \delta^{2} u_{0}$, 
or the mean of these, viz.,

$$
\text { (19) } u_{\theta}=\mu u_{3}-\left(\frac{1}{2}-\theta\right) \delta u_{1}+\frac{1}{2} \theta(\theta-1) \mu \delta^{2} \cdot u_{1} .
$$

(iii) If we have to go up to differences of an order higher than the 2nd, it will usually, as explained at the end of $\$ 5$, be found most convenient to express differential coefficients in terms of differences, and then use Taylor's theorem.

(a) For advancing differences the theoretically best result is obtained by having all differences after the 1st corrected, though practically (see Tahle VII below) we obtain nearly as good a result if those of even order only are corrected. In either case, we should use Taylor's theorem in the form given in $§ 5$, viz.,

$$
\begin{aligned}
u_{\theta} & =u_{0}+\theta h u_{n}^{\prime}+\frac{\theta^{2}}{2 !} h^{2} u_{0}^{\prime \prime}+\frac{\theta^{3}}{3 !} h^{3} u_{0}^{\prime \prime \prime}+\ldots \\
& \left.=u_{0}+\theta\left[h u_{n}^{\prime}+\frac{1}{2} \theta ! h^{2} u_{0}^{\prime \prime}+\frac{1}{3} \theta\left(h^{3} u_{0}^{\prime \prime \prime}+\ldots\right)\right\}\right] ;
\end{aligned}
$$

and the values of $h u_{0}^{\prime}, h^{2} u_{0}^{\prime \prime}, h^{3} u_{0}^{\prime \prime}, \ldots$, would be given by

$$
\begin{aligned}
& h u_{0}^{\prime}=\left(\Delta-\frac{1}{2} \Delta^{2}+\frac{1}{3} \Delta^{3}-\frac{1}{4} \Delta^{4}+\frac{1}{5} \Delta^{5}-\frac{1}{6} \Delta^{6}+\frac{1}{7} \Delta^{7}-\frac{1}{4} \Delta^{8}+\ldots\right) u_{0} \\
& h^{2} u_{0}^{\prime \prime}=\left(\Delta^{2}-\Delta^{3}+\frac{1}{1} \frac{1}{2} \Delta^{4}-\frac{5}{6} \Delta^{5}+\frac{1}{1} \frac{37}{8} \Delta^{6}-\frac{7}{10} \Delta^{7}+\frac{36}{5} \frac{3}{6} \Delta^{8}-\ldots\right) u_{0} \\
& h^{3} u_{n}^{\prime \prime \prime}=\left(\Delta^{3}-\frac{3}{2} \Delta^{4}+\frac{7}{4} \Delta^{5}-\frac{15}{K} \Delta^{6}+\frac{29}{15} \Delta^{7}-\frac{469}{2} \frac{9}{0} \Delta^{8}+\ldots\right) u_{0} \\
& h^{4} u_{0}^{\text {iv }}=\left(\Delta^{4}-2 \Delta^{5}+\frac{17}{6} \Delta^{6}-\frac{7}{2} \Delta^{7}+\frac{967}{2} \frac{7}{4} \Delta^{8}-\ldots\right) u_{0} \\
& h^{5} u_{0}^{v}=\left(\Delta^{5}-\frac{5}{2} \Delta^{6}+\frac{25}{6} \Delta^{7}-\frac{35}{6} \Delta^{8}+\ldots\right) u_{0} \\
& h^{6} u_{0}^{\mathrm{vi}}=\left(\Delta^{6}-3 \Delta^{7}+\frac{23}{4} \Delta^{8}-\ldots\right) u_{0} \\
& h^{7} u_{0}^{\mathrm{vil}}=\left(\Delta^{7}-\frac{7}{2} \Delta^{8}+\ldots\right) u_{0} \\
& h^{8} u_{0}^{u_{0}^{\text {iif }}}=\left(\Delta^{8}-\ldots\right) u_{0}
\end{aligned}
$$

We might in particular cases find it more convenient to use receding differences, working backwards from $u_{1}$, and making the necessary changes of sign of alternate columns of differences. The calculations of the differential coefficients, as shown by (86), are, however, more troublesome than the corresponding calculations for central differences.

(li) For central differences the stindard formula (16) has to be adapted 
in a similar way. One method of doing this has been explained in $\$ 5$; the formula (21) is converted into (25) by using the values of the differential coefficients as given by (26). It should, however, have been mentioned that insterd of (21) we might use (19). Writing $\theta \equiv \frac{1}{2}+\psi$, we obtain (23), which can be put in the form

$$
\begin{aligned}
& u_{\theta}=u_{\mathfrak{s}}+\psi h u_{3}^{\prime}+\frac{\psi^{2}}{2 !} h^{2} u_{\frac{3}{3}}^{\prime \prime}+\frac{\psi^{3}}{3 !} h^{3} u_{\frac{3}{3}}^{\prime \prime \prime}+\ldots \\
& =u_{i}+\psi\left[h u_{1}^{\prime}+\frac{1}{2} \psi\left\{h^{2} u u_{3}^{\prime \prime}+\frac{1}{3} \psi\left(h^{3} u u_{1}^{\prime \prime}+\ldots\right)\right\}\right] ;
\end{aligned}
$$

the values of $u_{b}, h u_{\mathfrak{k}}^{\prime}, h^{2} u_{\xi}^{\prime \prime}, h^{\mathrm{y}} u_{\underline{2}}^{\prime \prime \prime}, \ldots$ being given by*

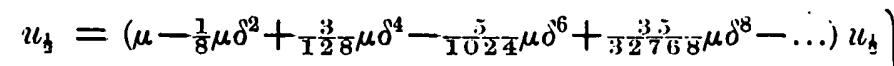

$$
\begin{aligned}
& h u u_{1}^{\prime}=\left(\delta-\frac{1}{24} \delta^{3}+\frac{3}{640} \delta^{5}-\frac{5}{716 \delta} \delta^{7}+\ldots\right) u_{\underline{1}} \\
& h^{2} u_{t}^{\prime \prime}=\left(\mu \delta^{2}-\frac{5}{24} \mu \delta^{4}+\frac{259}{5760} \mu \delta^{6}-\frac{3}{3} \frac{32}{2} \frac{29}{6} \sigma \mu \delta^{8}+\ldots\right) u_{3} \\
& h^{8} u_{1}^{\prime \prime \prime}=\left(\delta^{3}-\frac{1}{8} \delta^{5}+\frac{37}{9} \frac{7}{2} \delta^{7}+\ldots\right) u_{3} \\
& h^{4} u_{2}^{\mathrm{iv}}=\left(\mu \delta^{4}-\frac{7}{24} \mu \delta^{6}+\frac{47}{640} \mu \delta^{8}-\ldots\right) u_{\mathrm{k}} \\
& h^{5} u_{j}^{v}=\left(\delta^{5}-\frac{5}{24} \delta^{7}+\ldots\right) u_{k} \\
& h^{6} u_{\frac{1}{2 i}}^{\text {vi }}=\left(\mu \delta^{6}-\frac{3}{8} \mu \delta^{8}+\ldots\right) u_{\frac{1}{2}}
\end{aligned}
$$

As compared with (21), this method has, as will be seen from Tables III and IV, the theoretical advantage that it is immaterial whether we end with a difference of odd or of even order; but this advantage, as will be seen below, is very slight in comparison with the greater complication of the coefficients in (88). It is therefore only for values of $\theta$ very near $\frac{1}{2}$ that this method will be found useful. In ordinary cases we should use (21) ; taking care, with corrected differences, that the term in the limit of error which is due to the 2nd difference is $-\theta^{2} . \frac{1}{2} \rho$, not $+\frac{1}{2} \theta^{2} \cdot \frac{1}{2} \rho$, i.e., that we use $\left(21 \gamma^{\prime}\right),\left(21 \delta^{\prime}\right)$, or $(21 \epsilon)$.

The limits of error for the different methods, for formulæ which go up to differences not exceeding the 6 th, are shown for $\theta=\cdot 1, \cdot 2, \ldots, \cdot 9$ in the following table.

* Proc. Lundon Lllath. Soc., Vol. xxxr, p. 465, formulie (75). 
TABLE VII.

\begin{tabular}{|c|c|c|c|c|c|c|c|c|c|c|}
\hline \multirow{2}{*}{$\begin{array}{l}\text { Highest order } \\
\text { of differences } \\
\text { included in } \\
\text { formula. }\end{array}$} & \multirow{2}{*}{$\begin{array}{l}\text { Formula } \\
\text { and } \\
\text { system. }\end{array}$} & \multicolumn{9}{|c|}{ Limit of error $(\div \rho)$ for $\theta=$} \\
\hline & & $\cdot 1$ & $\cdot 2$ & $\cdot 3$ & $\cdot 4$ & $\cdot 5$ & $\cdot 6$ & $\cdot 7$ & $\cdot 8$ & $\cdot 9$ \\
\hline 3rd & $\begin{array}{c}(3 a) \\
(3 \gamma) \\
(3 \delta) \\
(16 a) \text { or }(19 a) \\
(16 \delta) \text { or }(19 \delta) \\
(21 a) \\
\left(21 \gamma^{\prime}\right) \text { or }\left(21 \delta^{\prime}\right) \\
(21 \epsilon)\end{array}$ & $\begin{array}{l}\cdot 631 \\
.537 \\
.551 \\
.545 \\
.523 \\
.570 \\
.554 \\
.554\end{array}$ & $\begin{array}{l}.724 \\
.564 \\
.588 \\
.580 \\
.540 \\
.628 \\
.596 \\
.596\end{array}$ & $\begin{array}{l}.784 \\
.583 \\
.612 \\
.605 \\
.553 \\
.674 \\
.628 \\
.628\end{array}$ & $\begin{array}{l}.812 \\
.592 \\
.624 \\
.620 \\
.560 \\
.704 \\
.648 \\
.648\end{array}$ & $\begin{array}{l}.813 \\
.594 \\
.625 \\
.625 \\
.563 \\
.719 \\
.657 \\
.657\end{array}$ & $\begin{array}{l}.788 \\
.588 \\
.616 \\
.620 \\
.560 \\
.716 \\
.652 \\
.652\end{array}$ & $\begin{array}{l}.742 \\
.576 \\
.598 \\
.605 \\
.553 \\
.695 \\
.635 \\
.635\end{array}$ & $\begin{array}{l}.676 \\
.556 \\
.572 \\
.580 \\
.540 \\
.652 \\
.604 \\
.604\end{array}$ & $\begin{array}{l}\cdot 595 \\
.531 \\
.539 \\
.545 \\
.523 \\
.588 \\
.560 \\
.560\end{array}$ \\
\hline 4 th & $\begin{array}{c}(3 a) \\
(3 \gamma) \\
(3 \delta) \\
(16 a) \text { or }(19 a) \\
(16 \delta) \text { or }(19 \delta) \\
(21 a) \\
\left(21 \gamma^{\prime}\right) \text { or }\left(21 \delta^{\prime}\right) \\
(21 \epsilon)\end{array}$ & $\begin{array}{l}.776 \\
.548 \\
.562 \\
.569 \\
.527 \\
.569 \\
.554 \\
.554\end{array}$ & $\begin{array}{l}.960 \\
.581 \\
.605 \\
.624 \\
.548 \\
.624 \\
.597 \\
.596\end{array}$ & $\begin{array}{r}1.065 \\
603 \\
633 \\
664 \\
.563 \\
.664 \\
630 \\
.628\end{array}$ & $\begin{array}{r}1 \cdot 104 \\
.613 \\
.645 \\
.688 \\
.572 \\
.688 \\
.651 \\
.648\end{array}$ & $\begin{array}{r}1 \cdot 086 \\
.614 \\
6445 \\
6996 \\
.575 \\
696 \\
661 \\
6657\end{array}$ & $\begin{array}{r}1.024 \\
.605 \\
.633 \\
.688 \\
.572 \\
.688 \\
.657 \\
.652\end{array}$ & $\begin{array}{l}.925 \\
.589 \\
.612 \\
.664 \\
.563 \\
.664 \\
.640 \\
.635\end{array}$ & $\begin{array}{l}.800 \\
.565 \\
.581 \\
.624 \\
.548 \\
.624 \\
.609 \\
.604\end{array}$ & $\begin{array}{l}.656 \\
.536 \\
.544 \\
.569 \\
.527 \\
.569 \\
.563 \\
.560\end{array}$ \\
\hline 5 th & $\begin{array}{c}(3 \alpha) \\
(3 \gamma) \\
(3 \delta) \\
(16 a) \text { or }(19 \alpha) \\
(16 \delta) \text { or }(19 \delta) \\
(21 \alpha) \\
\left(21 \gamma^{\prime}\right) \text { or }\left(21 \delta^{\prime}\right) \\
(21 \epsilon)\end{array}$ & $\begin{array}{r}1 \cdot 017 \\
.556 \\
.578 \\
.569 \\
.527 \\
.585 \\
.556 \\
.555\end{array}$ & $\begin{array}{r}1 \cdot 343 \\
.594 \\
631 \\
624 \\
.548 \\
655 \\
600 \\
6000\end{array}$ & $\begin{array}{r}1 \cdot 511 \\
.618 \\
.662 \\
.664 \\
.563 \\
.708 \\
.634 \\
.633\end{array}$ & $\begin{array}{r}1.553 \\
.628 \\
.675 \\
.688 \\
.572 \\
.741 \\
.657 \\
.654\end{array}$ & $\begin{array}{r}1 \cdot 497 \\
627 \\
672 \\
696 \\
.575 \\
.754 \\
6667 \\
663\end{array}$ & $\begin{array}{r}1 \cdot 366 \\
.617 \\
.656 \\
.688 \\
.572 \\
.746 \\
.663 \\
.658\end{array}$ & $\begin{array}{r}1 \cdot 184 \\
.597 \\
629 \\
6664 \\
.563 \\
.716 \\
640 \\
.640\end{array}$ & $\begin{array}{r}1 \cdot 469 \\
\cdot 571 \\
\cdot 593 \\
.624 \\
.548 \\
.664 \\
.613 \\
\cdot 609\end{array}$ & $\begin{array}{r}1 \cdot 236 \\
.538 \\
.549 \\
.569 \\
.527 \\
.592 \\
.565 \\
.562\end{array}$ \\
\hline 6th & $\begin{array}{c}(3 a) \\
(3 \gamma) \\
(3 \delta) \\
(16 a) \text { or }(19 a) \\
(168) \text { or }(19 \delta) \\
(21 a) \\
\left(21 \gamma^{\prime}\right) \text { or }\left(21 \delta^{\prime}\right) \\
(21 €)\end{array}$ & $\begin{array}{l}1 \cdot 425 \\
\cdot 562 \\
.585 \\
.585 \\
.528 \\
.585 \\
.556 \\
\cdot 555\end{array}$ & $\begin{array}{r}1 \cdot 976 \\
604 \\
6641 \\
653 \\
\cdot 549 \\
653 \\
601 \\
6000\end{array}$ & $\begin{array}{r}2 \cdot 233 \\
\cdot 629 \\
-674 \\
.704 \\
.565 \\
.704 \\
6035 \\
\cdot 633\end{array}$ & $\begin{array}{r}2 \cdot 265 \\
\cdot 640 \\
\cdot 687 \\
.734 \\
.574 \\
.734 \\
.657 \\
\cdot 654\end{array}$ & $\begin{array}{r}2 \cdot 132 \\
638 \\
683 \\
.745 \\
.577 \\
.745 \\
667 \\
663\end{array}$ & $\begin{array}{r}1.886 \\
.625 \\
.665 \\
.734 \\
.574 \\
.734 \\
.664 \\
.658\end{array}$ & $\begin{array}{r}1 \cdot 568 \\
.604 \\
.635 \\
.704 \\
.565 \\
.704 \\
646 \\
640\end{array}$ & $\begin{array}{r}1 \cdot 213 \\
.575 \\
.597 \\
.653 \\
.549 \\
.653 \\
.614 \\
.609\end{array}$ & $\begin{array}{r}1 \cdot 350 \\
\cdot 540 \\
\cdot 551 \\
\cdot 585 \\
\cdot 528 \\
\cdot 585 \\
\cdot 566 \\
\cdot 562\end{array}$ \\
\hline
\end{tabular}

It will be seen that the difference between $(3 \gamma)$ and (30) is very slight; and that there is not really much to choose between either of these and a central-difference formula with tabular or with corrected differences. If all the differences (after the 1st) are corrected, several courses are open to us. We may take the differences as they stand, using either $(3 \gamma)$ or $(19 \gamma)$ : we may replace the 2 nd difference by its tabular value, and use $\left(21 \gamma^{\prime}\right)$; we may, in addition to this, replace the 4th, 6th, ... differences by their semicorrected values, and use (21є); or, retaining the corrected values of the 2nd, 4th, 6th, ... differences, we may replace the 3rd, 5th, 7th, ... differences by their semi-corrected values, and use (19ס). If only differences of even order are corrected, we can use either $(3 \delta),(19 \delta)$, or $\left(21 \delta^{\prime}\right)$; re- 
membering that with $\left(21 \delta^{\prime}\right)$ the 2 nd difference actually used in the third term of the formula is to have its tabular value. The choice of a method, in any of these cases, is largely a matter of individual preference.

35. In the above investigation we have not taken into account the additional information with regard to the values of $u$ that is supplied by the corrected differences. The utilisation of this information for the purpose of improving the original table as a whole belongs rather to the theory of smoothing, but it is as well to see how its existence affects our formulæ.

(i) It should be noted in the first place that any smoothing that is done will be based rather on the differences of low order, and particularly on the 1st differences, thiun on those of high order. We can see this by reference to an individual case, such as the following :-

\begin{tabular}{|c|c|c|c|c|c|c|c|}
\hline$x$ & $\tan x$ & Tabula & diffe & ences. & Correcte & diffe & ences. \\
\hline $\begin{array}{l}46^{\prime \prime} \\
47^{\circ} \\
48^{\circ} \\
49^{\prime \prime} \\
50^{\circ} \\
51^{\circ} \\
52^{\circ}\end{array}$ & $\begin{array}{l}1 \cdot 03553 \\
1 \cdot 07237 \\
1 \cdot 110611 \\
1 \cdot 150: 37 \\
1 \cdot 191^{\prime} 15 \\
1 \cdot 23490 \\
1 \cdot 27994\end{array}$ & $\begin{array}{c}\Delta \\
+ \\
3684 \\
3824 \\
3976 \\
4138 \\
4315 \\
4504\end{array}$ & $\begin{array}{l}140 \\
152 \\
162 \\
177 \\
189\end{array}$ & $\begin{array}{l}12 \\
10 \\
15 \\
12\end{array}$ & $\begin{array}{c}\Delta \\
+ \\
3684 \\
3824 \\
3976 \\
4139 \\
4314 \\
4504\end{array}$ & $\begin{array}{l}141 \\
151 \\
163 \\
176 \\
190\end{array}$ & $\begin{array}{l}11 \\
12 \\
13 \\
14\end{array}$ \\
\hline
\end{tabular}

Here, although the contrist between the tabular and the corrected differences is less striking for the 1st differences than for the 3rd differences, it is really more important for the former than for the latter; for we could easily obtain the more accurate differences of the higher order either by smoothing the tabular differences or (which comes to very much the same thing) by working from a table in which the intervals of $x$ are greater.

(ii) Further, we cannot alter the determining values of one interval, in any scientific manner, without considering them in relation to the adjoining values, i.e., without considering the series of values of $u$ as a whole; and that is too complicated a matter to be considered here. It is true that, in the particular example, the two corrected 1st differences which differ from the tabular differences are consecutive, so that we might adjust the table by a guess-work alteration of $\tan 50^{\circ}$; but that is an accident. We must therefore ussume that we start with the values of $u$ as given in the table. 
(iii) Now consider the case of corrected 1st differences. With the notation of $\$ 2$, the errors in the tabulation of $u_{0}$ and of $u_{1}$ are $\alpha_{0}$ and $\alpha_{1}$, the tabulated values being $U_{0} \equiv u_{0}+\alpha_{0}$ and $U_{1} \equiv u_{1}+\alpha_{1}$. The true value of $u_{1}-u_{0}$ is therefore $U_{1}-U_{0}-\left(\alpha_{1}-a_{0}\right)$. The tabular 1st difference of $u_{0}$ and $u_{1}$ is $U_{1}-U_{0}$; and the corrected 1st difference will be $U_{1}-U_{0}+\rho$, $U_{1}-U_{0}$, or $U_{1}-U_{0}-\rho$, according as $\alpha_{1}-a_{0}$ lies between $-\rho$ and $-\frac{1}{2} \rho$, between $-\frac{1}{2} \rho$ and $+\frac{1}{2} \rho$, or between $+\frac{1}{2} \rho$ and $+\rho$.

(a) Taking the second case first, suppose that the tabular difference und the corrected difference are the same. Then, although we have some information as to the value of $\alpha_{1}-a_{0}$, we have really no more information as to the value of

$$
u_{0}+\theta\left(a_{1}-\alpha_{0}\right) \equiv(1-\theta) a_{0}+\theta a_{1}
$$

than we should have had if we had been dealing with a table showing tabular differences only; for the data are quite consistent with the possibility of both $U_{0}$ and $U_{1}$ having a large error in the same direction. It might, for instance, be the case that $\alpha_{0}=a_{1}=499 \rho$, and this would then be also the value of $a_{0}+\theta\left(a_{1}-a_{0}\right)$. The knowledge that the tabular difference and the corrected difference are the same does not therefore enable us to reduce the limit of error.

(b) Next, suppose that, as in the interval $\left(49^{\circ}, 50^{\circ}\right)$ in the above example, $a_{1}-a_{0}$ lies between $-\rho$ and $-\frac{1}{2} \rho$, so that the corrected 1st difference exceeds the tabular 1st difference by $\rho$.

(1) We note in the first place that this means that $a_{0}$ is positive and $\alpha_{1}$ negative, so that for interpolation towards the middle of the interval the errors will tend to counteract each other. This also applies if $\alpha_{1}-a_{0}$ lies between $+\frac{1}{2} \rho$ and $+\rho$. It is therefore in those cases in which the tabular and the corrected difference are different that the ordinary formula, using the tabular difference, produces a specially good result.

(2) Now consider the actual limits of error of the result of the ordinary formula, using the corrected difference. Writing $\alpha_{1} \equiv-\alpha_{1}^{\prime}$, so that $a_{0}$ and $u_{1}^{\prime}$ are both positive, we shall have as the result of the formula (differences after the 1st being ignored)

$$
\begin{aligned}
u_{\theta}=U_{0}+\theta\left(U_{1}-U_{0}+\rho\right) & =u_{0}+\theta\left(u_{1}-u_{0}\right)+u_{0}+\theta\left(-\alpha_{1}^{\prime}-u_{0}+\rho\right) \\
& =u_{0}+\theta\left(u_{1}-u_{0}\right)+(1-\theta) u_{0}-\theta u_{1}^{\prime}+\theta \rho ;
\end{aligned}
$$


and this will lie between

$$
u_{0}+\theta\left(u_{1}-u_{0}\right)+\theta \cdot \frac{1}{2} \rho \text { and } u_{0}+\theta\left(u_{1}-u_{0}\right)+(1+\theta) \frac{1}{2} \rho .
$$

Hence the limits of error, due to using the corrected difference, are $+\theta \cdot \frac{1}{2} \rho$ and $+(1+\theta) \frac{1}{2} \rho$.

(c) Similarly, if the corrected 1st difference is less than the tabular 1st difference, so that $\alpha_{0}$ is shown to be negative and $\alpha_{1}$ positive, the limits of error, due to using the corrected difference, are $-(1+\theta) \frac{1}{2} \rho$ and $-\theta \cdot \frac{1}{2} \rho$.

(d) Comparing the three cases, we see that, while the error for 1stdifference interpolation with corrected difference may reach $-R$ on the negative side, and may reach $+R$ on the positive side, where [see $\$ 27$ ] $R=(1+\theta) \frac{1}{2} \rho$, it will never in any particular case have so great a range as from $-R$ to $+R$. In one of the three classes of possible cases its range will be from $-\frac{1}{2} \rho$ to $+\frac{1}{2} \rho$, in another from $R-\frac{1}{2} \rho$ to $R$, and in the third from $-R$ to $-R+\frac{1}{2} \rho$.

(e) Further, while the length of the range in the first class of cases is $\rho$ (as it is whenever we use the tabular difference), in the other two it is only $\frac{1}{2} \rho$. Hence we can reduce the limit of error in these latter cases to $\pm \frac{1}{4} \rho$ by using a formula which is midway between the two limiting values. We should therefore, if the corrected 1st difference is greater than the tabular 1st difference, take

$$
\begin{aligned}
u_{\theta} & =U_{0}+\theta\left(U_{1}-U_{0}\right)+\left(\theta-\frac{1}{2}\right) \frac{1}{2} \rho \\
& =\left(U_{0}-\frac{1}{4} \rho\right)+\theta\left\{\left(U_{1}+\frac{1}{4} \rho\right)-\left(U_{0}-\frac{1}{4} \rho\right)\right\} ;
\end{aligned}
$$

and, if the corrected 1st difference is less than the tabular 1st difference,

$$
\begin{aligned}
u_{\theta} & =U_{0}+\theta\left(U_{1}-U_{0}\right)-\left(\theta-\frac{1}{2}\right) \frac{1}{2} \rho \\
& =\left(U_{0}+\frac{1}{4} \rho\right)+\theta\left\{\left(U_{1}-\frac{1}{4} \rho\right)-\left(U_{0}+\frac{1}{4} \rho\right)\right\} ;
\end{aligned}
$$

and in each case the limits of resulting error will be $\pm \frac{1}{4} \rho$. We might have obtained these results more directly from the information given as to the signs of $\alpha_{0}$ and $\alpha_{1}$. In the first of the two cases, for instance, $\alpha_{0}$ is positive and $\alpha_{1}$ negative, so that $u_{0}$ lies between $U_{0}-\frac{1}{2} \rho$ and $U_{0}$, and $u_{1}$ between $U_{1}$ and $U_{1}+\frac{1}{2} \rho$; and therefore by replacing $U_{0}$ and $U_{1}$ by $U_{0}-\frac{1}{4} \rho$ and $U_{1}+\frac{1}{4} \rho$, we have values which are within $\pm \frac{1}{4} \rho$ of the true values, and the limits of error for $u_{\theta}$, if we interpolate by means of the tabular difference of these, will also be $\pm \frac{1}{4} \rho$.

(iv) It would be possible to extend this method to differences of the 
2nd and higher orders, and to lay down rules for adjusting the values of $u$ (within the tabular triangle of the particular formula) by means of the tabular differences in such a way as to enable us to make further reductions in the limits of error of the formula. The number of different cases would, however, increase considerably; and, unless we set to work to smooth the table as a whole, it seens simplest to confine the method to 1st differences. We can, of course, after having adjusted any values of $u$, continue with corrected differences as they appear in the table; the terms in the general expression for the limit of error, which are due to the terms involving these differences in the formula, will not be affected by the reduction (by $\frac{1}{4} \rho$ ) of the portion due to the first two terms.

\section{The Arrangement of Mathematical Tables for Interpolation.}

36. The question then arises whether, in setting out a mathematical table, the tabular or the corrected differences should be given.

(i) The entry of the corrected differences does not involve any additional trouble, except as regards a limited number of values. To obtain a table to (say) 7 places of decimals, we must work to 9 or 10 places and must check our results by differencing; and we can correct the final figures of the differences in the same way that we correct those of the u's. The only question is with regard to those cases in which the corrected tinal tigure of the difference is doubtful. To make the table perfect, all such cases should be specially treated, and this certainly increases the labour, especially if we go to differences of a high order. But in practice it is hardly worth while doing this, since the portions of the limit of error which are due to terms involving high differences are relatively small. A table in which all the values of $u$ are properly corrected in the final figure, and the limits of error of the differences are stated, may be regarded as a good table.

(ii) As regards appearance, each kind of table has its disadvantages, but these are largely a matter of habit. On the corrected-difference system, the sequence of differences of a high order is more regular, but the discrepancies between values in successive columns may be disturbing.

(iii) 'The special advantage of the corrected-difference system is that it 
gives additional information. But it has been shown $[\S 35]$ that, so far as the tabulated values are concerned, important information is only given in those cases where the tabular and the corrected 1st differences are different, and $[\S 34]$ that, so far as interpolated values are concerned, the increase of accuracy due to corrected differences above the second, though appreciable, is not very great, if we use central-difference formulæ.

(iv) Moreover, we have found that, where the corrected 1st difference gives information as to the values of the $u$ 's, it only does so indirectly, by showing the signs of the errors. This information might be given directly, not merely for these particular $u$ 's but for all the $u$ 's in the table, by the system, sometimes adopted, of marking the final figure in those cases in which it has been increased. By paying attention to these marks we can at once reduce the limit of error of each $u$ from $\pm \frac{1}{2} \rho$ to $\pm \frac{1}{4} \rho$.

(v) On the other hand, the system of tabular differences, besides being simpler in itself, has the special advantage of enabling us to check printers' errors, and also, in the preparation of the table for printing, to check errors of transcription. This, however, applies mainly to pairs of successive columns, and we should equally be able to check printers' errors if we had correctel and semi-corrected differences alternately.

(vi) It should be remembered that there are really two classes of mathematical tables, viz., those intended for general use, und those that will be used mainly by the specialist. In the former, the number of tabulated values is as great as possible, so as to avoid the necessity of using differences of a high order, while yet retaining a greater degree of accuracy in the tabulated values than will ordinarily be required; in the latter, considerations of economy prevent the preparation and publication of very full tables, the degree of accuracy of such tables as are published is often barely sufficient, and there is not the same objection to special methods of tabulation.

(vii) On the whole, the conclusion seems to be that in tables intended for general use, and not going beyond (say) 2 nd differences, it is best to use tabular differences throughout; but that in special tables, where we go up to differences of a relatively high order, it is best to give the corrected velues of the differences of even order, the tabular 1st clifferences, and the semi-corrected values of the remainder, and to mark those values 
of $u$ in which the final figure has been altered by correction, i.e., in which the tabular error of $U$ is positive.

(viii) It is, of course, necessary that in all cases the degree of accuracy should be correctly stated, either directly or by implication. If no such statement is made, it is to be assumed that the finel figure, where it professes to be corrected, is really properly corrected. Unfortunately, it cannot be certain that this is always done; there are probably a good many tables in existence which are ostensibly correct in the final figures, but in which the values have not been specially tested in doubtful cases. 